\title{
A Homogenized Free Energy Model for Hysteresis in Thin-film Shape Memory Alloys
}

\author{
Jordan E. Massad ${ }^{1}$ and Ralph C. Smith \\ Center for Research in Scientific Computation, N.C. State Univ., Raleigh, NC \\ 27695-8205
}

\begin{abstract}
Thin-film shape memory alloys (SMAs) have become excellent candidates for microactuator fabrication in MEMS due to their capability to achieve very high work densities, produce large deformations, and generate high stresses. In general, the material behavior of SMAs is nonlinear and hysteretic. To achieve the full potential of SMA actuators, it is necessary to develop models that characterize the nonlinearities and hysteresis inherent to the constituent materials. We develop a model that quantifies the nonlinearities and hysteresis inherent to SMAs. The model is based on free energy principles combined with stochastic homogenization techniques. The fully thermomechanical model predicts rate-dependent, polycrystalline SMA behavior, and it accommodates heat transfer issues pertinent to thin-film SMAs. We illustrate aspects of the model through comparison with thin-film SMA superelastic and shape memory effect hysteresis data.
\end{abstract}

Key words: Shape memory alloy model; thin film; polycrystals.

\section{Introduction}

Shape memory alloys (SMAs) are metals that recover from up to $10 \%$ deformation via stress and temperature-induced phase transformations. SMAs undergo martensitic transformations, which are displacive transformations dominated by shear distortions of the crystal lattice. Transformations occur between solid phases, termed the martensite and austenite phases. Distinguished by their crystallographic structures, martensite and austenite can have drastically different mechanical, thermal, electrical, optical, and acoustical material properties [82]. In general, martensite is the material phase that is stable at low temperatures relative to austenite, which is stable at high temperatures.

$\overline{1}$ Corresponding author; Email: jemassa@sandia.gov; Tel.: 505-844-2952. 
The martensitic transformations between martensite and austenite enable SMAs to recover or "remember" shape by two different mechanisms. In both cases, austenite corresponds to the remembered shape. First, superelasticity describes shape memory via stress-induced phase transformations. At a fixed temperature where stress-free austenite is a stable phase, austenite transforms into martensite due to an applied load. Upon removing the load, the material reverts to austenite and the original shape is recovered. Secondly, the shape memory effect (SME) describes shape memory via temperature-induced transformations. In this case, deformed martensite transforms into austenite due to heating and shape memory is observed. Upon subsequent cooling, the SMA transforms back to martensite. If the SMA is stress-free upon cooling, then it will retain its recovered shape in the martensite phase by means of a process called self-accommodation. If the SMA is subjected to a load while cooling, then it will deform again as it reverts to martensite.

As illustrated in Figure 1, hysteresis is associated with both superelasticity and the shape memory effect. For superelasticity, it is observed that austenite deforms elastically until a loading transformation is reached. Further loading induces a transformation to the martensite phase with a large transformation strain. Upon unloading martensite, the transformation strain is recovered as the SMA returns to austenite. For the shape memory effect, hysteresis is often observed when an SMA is subjected to a fixed load. In this case, an austenitic SMA will transform to martensite when cooled, exhibiting a large transformation strain in the process. When reheated to austenite, the transformation strain is recovered. The general stress-temperature-strain behavior of SMAs is thermomechanically coupled. We refer the reader to [82] for details of shape memory mechanisms and other SMA material properties.

At the heart of the first-order martensitic phase transitions in SMAs are displacive phase transitions at the crystal lattice. NiTi and many Cu-based SMAs admit a cubic crystal structure in the high-temperature austenite phase. Upon
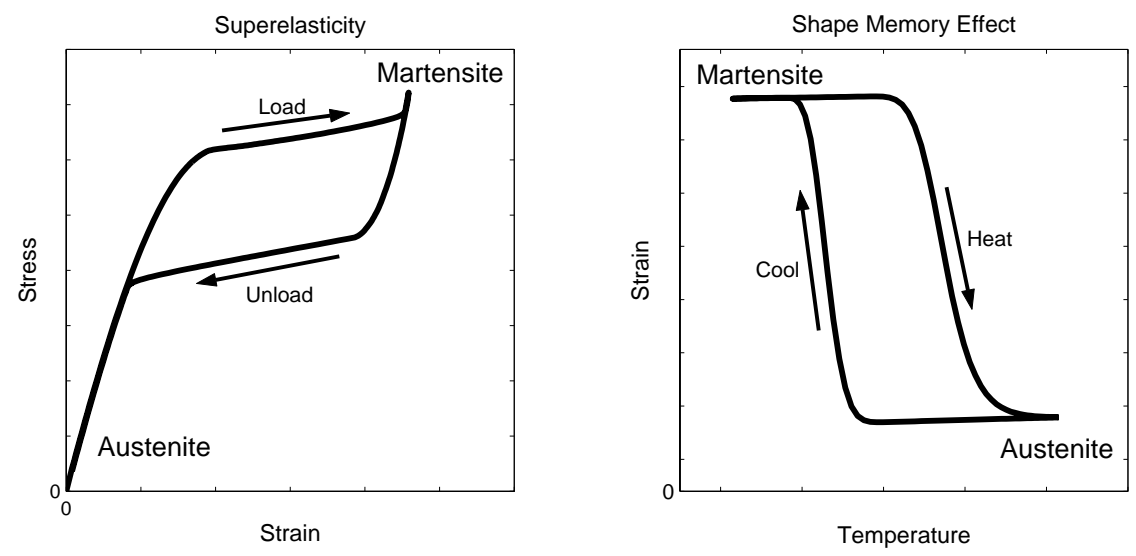

Fig. 1. Hysteresis associated with superelasticity (fixed temperature) and the shape memory effect (fixed load). 
transformation to martensite, the high-symmetry structure decomposes into a lower-symmetry, martensite structure. The symmetry reduction allows for several differently-oriented martensite single crystals to form from a single austenite crystal via predominantly shear lattice distortions. We refer the reader to $[12,34,82]$ for greater detail on SMA crystallography.

We note that with particular material preparation, the transformation from austenite to martensite (cooling or stress-induced) involves an intermediate rhombohedral phase with a trigonal crystal lattice. The shape change associated with the intermediate transformation is on the order of $0.1 \%$ and its thermal hysteresis is an order smaller than that of the direct austenite-martensite transformation. The rhombohedral phase can usually be eliminated with heat treatment [82]. For our one-dimensional model, we focus on direct transformations between austenite and martensite and we consider a simplified case with only two orientations of martensite, denoted $M^{+}$and $M^{-}$, that are sheared versions of austenite $(A)$.

Since their discovery in 1932, SMAs have been fabricated mostly in the form of wires, tubes, and bars. However, in the last 10 years, shape memory alloys have been fabricated as thin foils and films. To make the batch production of thin-film SMA devices viable, research is ongoing to develop thin-film SMA fabrication techniques that are compatible with conventional integrated circuit processing and bulk micromachining. The predominant fabrication method for SMA films has been sputter deposition (RF and DC magnetron) $[38,29,48,75,78,82]$. SMA films differ from bulk SMAs in several aspects, including their structure (e.g., very fine-grained) and their shape memory behavior. Moreover, an increased sensitivity of material properties to chemical composition, impurities, and processing conditions is observed in SMA films $[28,48,54,109,117]$. In the following section, we discuss applications of SMAs that have resulted from advances in thin-film processing.

\subsection{Applications}

The hysteresis exhibited by shape memory alloys enables the materials to achieve very high work densities, produce large recoverable deformations, and generate high stresses, which are ideal for a number of high performance applications. For example, SMA wires have been considered for medical and potential aeronautic and aerospace applications that require large deformations and large forces $[22,52]$. Additionally, SMAs exhibit a damping capacity much larger than that of a number of conventional materials. In this case, SMA hysteresis is being utilized to design earthquake and hurricane-resistant civil structures $[21,92,105,113]$. Most of these macroscopic applications are limited in bandwidth because of heating and cooling restrictions inherent to bulk SMAs. 
Recent development of SMA films has made SMAs excellent candidates for microactuators. Their work output density is on the order of $10 \mathrm{MJ} \mathrm{m}^{-3}$, which exceeds that of other microactuator materials [78,116]. Additionally, they can yield large strokes and forces on the order of $1 \%$ and $100 \mathrm{mN}$, respectively. Moreover, thin-film SMAs heat on the order of milliseconds by low voltage Joule heating ( $\sim 5 \mathrm{~V})$, and, unlike macroscopic SMAs (wires, tubes, bars, etc.), their small thermal mass and large surface-to-volume ratio allow fast cooling rates, potentially permitting switching frequencies on the order of $100 \mathrm{~Hz}$ $[27,73,87,97]$.

Microscale in-plane applications include microgrippers with large grip sizes and strengths $[9,10,44,59,70,90,110,121]$, high current carrying, latching microrelays [32], microswitches [10], microshutters [60], and loop actuators [30]. Several thin-film SMA microcantilevers have been fabricated for applications that require out-of-plane actuation $[29,49,56,65,66,79,118]$. In particular, NiTi microcantilevers are used in [40] for far infrared imaging at ambient temperatures where SMA cantilevers deflect in response to small temperature changes caused by radiation absorption.

Thin-film SMA strips have been investigated for self-propelled, low power microrobots [114], and to generate traveling waves for turbulent drag reduction [115]. Moreover, thin-film SMAs in the form of membranes have been considered for micropumps $[11,74,119]$ and microvalves $[57,58]$ that generate large pressures and pumping rates. In [98], a thin-film SMA membrane is used in a prototype compact hybrid actuator to reach drive frequencies of $100 \mathrm{~Hz}$ and output forces exceeding $100 \mathrm{~N}$. In [104], the change in optical reflectivity from austenite to martensite is utilized to fabricate a membrane-based light-valve.

Many of these applications rely on the one-way memory effect and require an extrinsic biasing mechanism for full actuation. However, microdevices using functionally graded films [37,38] or bimorph principles $[29,43,49,66,79,102]$ can achieve two-way, out-of-plane displacements with smaller footprints than conventional micromechanisms. Finally, superelastic NiTi thin films are being considered for high-strength surface coatings in MEMS devices, and they have potential for microscale mechanical energy storage devices, and vibration dampeners in microelectronics packaging $[39,42]$. High damping has also been observed in multilayer SMA film microcantilevers [20]. We refer the reader to $[18,48,63]$ for reviews of other SMA microscale applications. 


\subsection{Modeling Approaches}

To achieve the full potential of SMA actuators, it is necessary to develop models that characterize the nonlinearities and hysteresis inherent in the materials. Most models of hysteresis in SMAs are constitutive, aiming to predict the measured relationships among stress, temperature, and strain. We refer the reader to reviews and comparisons of a number of models in $[15,17,25,35,88]$ and particularly in [93], where computational considerations are addressed. SMA hysteresis models can be roughly categorized as being microscopic, mesoscopic, or macroscopic, depending on which material level they base their method of predicting constitutive behavior.

Microscopic and mesoscopic models, such as $[6,7,19,120]$, employ both phenomenological and energy principles to model atomic and lattice-level behavior of ferroelastic compounds. Understanding material dynamics at these fundamental levels can support efforts to design compounds with desired material properties. For example, Castán, et. al. [19] quantify interatomic energies and conduct lattice model simulations for some ferroelastic alloys. Given atomic composition and thermal treatment information, they are able to compute macroscopic elastic constants and martensite transition temperatures. Models of this nature are typically used for off-line simulations, and their solution requires techniques such as Monte Carlo methods that have a high computational cost.

Another class of mesoscopic models, traditionally referred to as micromechanical models, focuses specifically on developing local grain-level constitutive theories $[24,34,84]$. While operating at a fundamental level similar to that of the previously mentioned theory, these models provide a more direct means of predicting observed constitutive behavior. Deriving macroscopic constitutive behavior from these theories for design applications necessitates additional procedures, such as the self-consistent averaging approaches in [34,84]. Scaling these mesoscopic theories to macroscopic levels usually is computationally intensive; therefore, for macroscopic predictions, these models are generally not intended for on-line engineering nor control applications. Recently, micromechanical models have been developed specifically for thin-film SMAs $[8,13,53,99]$.

Macroscopic models commonly employ phenomenological or energy principles. As opposed to most microscopic and mesoscopic models, macroscopic models are formulated mainly for implementation into engineering designs and on-line control. A series of internal-state models rooted in the uniaxial Tanaka approach $[15,88]$ use an empirical or thermodynamics-based evolution law for the martensite volume fraction, which in-turn is used to predict stress or strain using a phenomenological constitutive relation. Similarly, Papenfuß and Seelecke $[83,94]$ predict thermomechanical behavior by modeling the evolution 
of martensite variant fractions, but by using a statistical thermodynamics description of thermally activated processes. Lexcellent, et.al. [31,71] have developed similar models for thin-film SMAs.

Another macroscopic approach, based on phenomenological principles, is the Preisach model [108]. Originally developed for ferromagnetic hysteresis, Preisach models have been generalized and adapted to other physical systems, including SMAs $[33,45,46,64,67,77,112]$. In general, Preisach models are purely empirical and their implementation reduces to the identification of many mathematical parameters via numerous hysteresis experiments that may be unavailable in practice. To make Preisach models more tractable for SMA applications, there have been attempts to replace or identify purely mathematical constructs with known or approximated physics. For example, Huo [46] incorporates Falk's [23] macroscopic Landau-Ginzburg potential to account for first-order martensitic phase transformations. In addition, Lagoudas and Bhattacharyya [67] associate Preisach weighting functions with distributions of single-crystal orientations in polycrystalline SMAs.

The model we present focuses on predicting macroscopic constitutive behavior by starting with mesoscopic energy relations. The model quantifies constitutive nonlinearities and hysteresis inherent to SMAs in formulations suitable for subsequent model-based control design. We employ as a starting point the Müller-Achenbach-Seelecke theory [3,83,94], based on the quantification of thermally activated processes for bulk SMAs. In the first step, we establish local free energies for single-crystal, homogeneous SMAs and we employ transition state theory to derive rate laws for phase fraction evolution. In addition, we formulate a balance of internal energy that quantifies a rate-dependent release of latent heats and heat transfer to and from the environment. In the second step of the development, we extend the single-crystal model to accommodate inhomogeneous and polycrystalline materials by considering material parameters to be manifestations of underlying stochastic distributions. The result is a rate-dependent, thermomechanical model that predicts relative elongation due to applied stress and temperature. The model accommodates SMA behavior pertinent to actuator design, such as superelasticity and the shape memory effect. Moreover, the model admits a low-order formulation suitable for subsequent control design, and most of the model parameters are identifiable directly from standard measurements. In Section 2, we develop the uniform lattice model, and we extend the model to accommodate inhomogeneous and polycrystalline SMAs in Section 3. In Section 4, we address numerical implementation issues and analyze model simulations. Finally, in Section 5 we discuss the identification of material parameters and in Section 6 we validate aspects of the model through comparison with hysteresis data from and SMA foil and thin films. 


\section{Uniform Lattice Model}

Motivated by the theory in $[83,94]$, we treat a lattice layer as the fundamental element of our model. Following our simplified uniaxial description, we assume an SMA lattice layer admits either the austenite phase or one of two martensite variants. We denote the volume fraction of austenite and martensite layers in an SMA as $x_{\alpha}(t)$, where the subscript $\alpha$ denotes austenite $(A)$ and martensite (one of two opposing variants $M^{ \pm}$). The phase fractions satisfy the conservation law

$$
\sum_{\alpha} x_{\alpha}(t)=1
$$

over all time $t>0$. Throughout, we assume the martensite variants share the same thermomechanical properties, which generally differ from those of austenite. For specific heat capacities, we denote the volumetric quantity $c=\rho c_{V}$ for mass density $\rho$ and specific heat $c_{V}$ measured at constant volume. Once we construct a thermoelastic free energy relation for SMAs, we will model the evolution of the phase fractions as a function of stress and temperature.

\subsection{Energy Relations}

In this section, we construct a phenomenological description of an SMA's local free energy. First we construct the local Helmholtz and Gibbs free energies from elastic and thermodynamics relations based on the theory in [1,94]. Then, we describe equilibrium stress-strain equations and transformation behavior based on our energy expressions.

\subsubsection{Helmholtz Free Energy}

For a scalar strain $\varepsilon$, we consider the potential

$$
\phi_{ \pm}(\varepsilon, T)=\frac{E_{M}}{2}\left(\varepsilon \mp \varepsilon_{T}\right)^{2}+\beta_{M}(T)
$$

for the martensite variants, whereas for austenite we consider

$$
\phi_{A}(\varepsilon, T)=\frac{E_{A}}{2} \varepsilon^{2}+\beta_{A}(T)
$$

The strain-dependent portions of the potentials represent the elastic energies, where the constants $E_{M}$ and $E_{A}$ are linear elastic moduli for martensite and austenite, respectively. The quantity $\varepsilon_{T}$ corresponds to the stress-free equilibrium strain of martensite, while $\varepsilon=0$ (no deformation) is the equilibrium strain for austenite. The parameter-dependent family of functions $\beta_{\alpha}(T)$ represent the chemical (non-elastic) free energies, which we define in Section 2.1.3. 
While others, such as $[5,31,53,68,71]$, have formulated the specific Helmholtz free energies as a combination or mixture of (2) and (3), we construct a single, $\mathcal{C}^{1}$-continuous Helmholtz free energy by joining the individual potentials. We employ the Helmholtz relation

$$
\psi(\varepsilon, T)=\left\{\begin{array}{cc}
\frac{E_{M}}{2}\left(\varepsilon+\varepsilon_{T}\right)^{2} & \varepsilon \leq-\gamma_{M}(T) \\
-\frac{E_{0}(T)}{2}\left(\varepsilon+\varepsilon_{0}(T)\right)^{2}+\psi_{0}(T)-\gamma_{M}(T)<\varepsilon<-\gamma_{A}(T) & |\varepsilon| \leq \gamma_{A}(T) \\
\frac{E_{A}}{2} \varepsilon^{2}+\Delta \beta(T) & \gamma_{A}(T)<\varepsilon<\gamma_{M}(T) \\
-\frac{E_{0}(T)}{2}\left(\varepsilon-\varepsilon_{0}(T)\right)^{2}+\psi_{0}(T) & \varepsilon \gamma_{M}(T) \\
\frac{E_{M}}{2}\left(\varepsilon-\varepsilon_{T}\right)^{2} & 1
\end{array}\right.
$$

where $\gamma_{M}(T)$ and $\gamma_{A}(T)$ are temperature-dependent nodes connecting the concave parabolae, which represent unstable states, to the convex potentials, which represent stable martensite and austenite states. In (4), we have neglected elastic energies due to thermal expansion effects, which we introduce in Section 2.4. Without loss of generality, we have shifted the martensite minima to zero so that the austenite minimum has the height

$$
\Delta \beta(T)=\beta_{A}(T)-\beta_{M}(T) .
$$

When $\Delta \beta(T)<0$, the austenite equilibrium has a lower energy, so transformations from martensite to austenite are more likely to occur. The temperaturedependent coefficients $E_{0}, \varepsilon_{0}$, and $\psi_{0}$ define the concave parabolae whose maxima are $\psi_{0}$ at $\varepsilon= \pm \varepsilon_{0}$. Enforcing continuity at the nodes yields

$$
-E_{A} \gamma_{A}(T) \gamma_{M}(T)-E_{M}\left(\gamma_{M}(T)-\varepsilon_{T}\right)\left(\varepsilon_{T}-\gamma_{A}(T)\right)=2 \Delta \beta(T),
$$

which relates the temperature-dependence of the nodes to the chemical free energy. In the same manner, we obtain

$$
\begin{gathered}
E_{0}(T)=\frac{E_{A} \gamma_{A}(T)-E_{M}\left(\gamma_{M}(T)-\varepsilon_{T}\right)}{\gamma_{M}(T)-\gamma_{A}(T)} \\
\varepsilon_{0}(T)=\gamma_{A}(T) \frac{E_{A} \gamma_{M}(T)-E_{M}\left(\gamma_{M}(T)-\varepsilon_{T}\right)}{E_{A} \gamma_{A}(T)-E_{M}\left(\gamma_{M}(T)-\varepsilon_{T}\right)} \\
\psi_{0}(T)=-\frac{1}{2} \frac{E_{M}\left(\gamma_{M}(T)-\varepsilon_{T}\right)\left[E_{A} \gamma_{A}(T) \varepsilon_{T}+2 \Delta \beta(T)\right]}{E_{A} \gamma_{A}(T)-E_{M}\left(\gamma_{M}(T)-\varepsilon_{T}\right)} .
\end{gathered}
$$

Note that the local three-well energy formulation (4) is only valid at temperatures for which austenite is stable. We define a local critical temperature $T_{M}$ below which the austenite phase is unstable upon cooling for a single lattice 

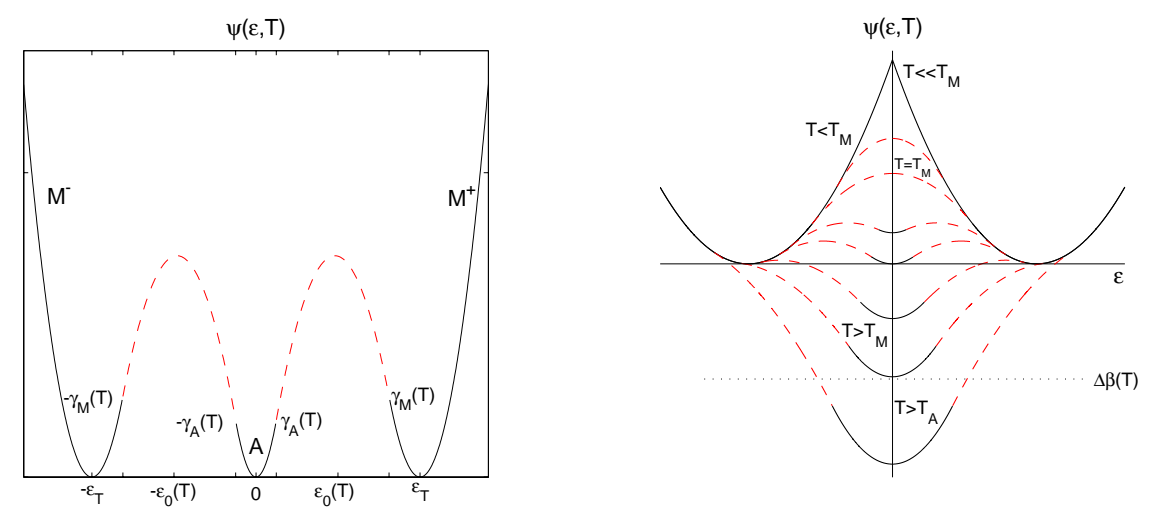

Fig. 2. Piecewise quadratic Helmholtz free energy density (4) and (10) for increasing temperatures. Dashed segments represent the concave, unstable states and $\Delta \beta(T)$ is the height of the austenite minimum.

layer. Then, (4) holds only for $T \geq T_{M}$ and the austenite nodes $\gamma_{A}(T)$ converge to zero at $T=T_{M}$. In practice, superelastic and SME behavior occur at temperatures sufficiently greater than $T_{M}$; however quasiplastic and low-stress thermoelastic applications achieve complete actuation at lower temperatures. For $T \leq T_{M}$, we model the Helmholtz free energy as the double-well potential

$$
\psi(\varepsilon, T)=\left\{\begin{array}{cc}
\frac{E_{M}}{2}\left(\varepsilon+\varepsilon_{T}\right)^{2} & \varepsilon \leq-\gamma_{M}(T) \\
\frac{-\Delta \beta(T)}{\gamma_{M}(T) \varepsilon_{T}} \varepsilon^{2}+\Delta \beta(T) & |\varepsilon|<\gamma_{M}(T) \\
\frac{E_{M}}{2}\left(\varepsilon-\varepsilon_{T}\right)^{2} & \varepsilon \geq \gamma_{M}(T)
\end{array}\right.
$$

with

$$
E_{M} \varepsilon_{T}\left(\varepsilon_{T}-\gamma_{M}(T)\right)=2 \Delta \beta(T)
$$

Since $\gamma_{M}(T) \geq 0$ by construction, (11) provides the restriction $\Delta \beta(T) \leq$ $E_{M} \varepsilon_{T}^{2} / 2$. This restriction is imposed by our choice of piecewise functions and it implies that the calculated chemical free energy difference cannot exceed the maximum transformation energy for phase transformations between martensite variants. Note that the higher-order potentials developed in $[1,2,23,76]$ do not have this limitation. Provided $\Delta \beta(T)$ reaches this limiting value, it will occur for temperatures below $T_{M}$ where the martensite nodes converge to zero.

Additionally, there is a temperature $T_{A}>T_{M}$ such that for temperatures $T>T_{A}$, there are no martensite equilibria. For a single layer, $T>T_{A}$ corresponds to the high-temperature austenite state. Figure 2 illustrates (4) and (10) for the full range of temperatures assuming $\frac{\partial \Delta \beta}{\partial T}<0$. 

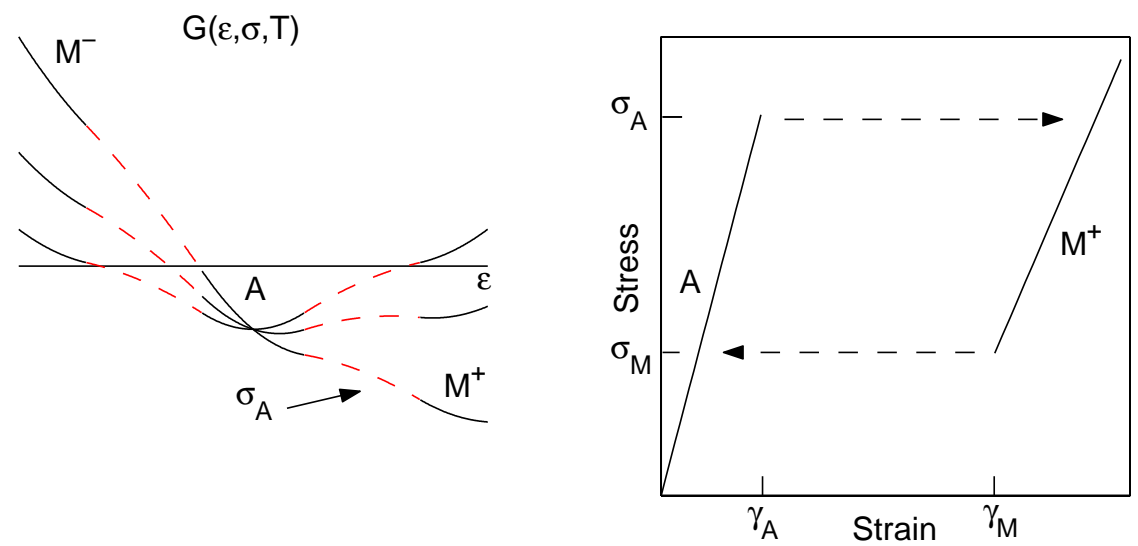

Fig. 3. The specific Gibbs free energy (12) at a fixed temperature $T>T_{M}$ and varying stress. The equilibrium conditions (13) yield a hysteretic stress-strain relation with jumps at the nodes.

\subsubsection{Gibbs Free Energy}

A Gibbs free energy density, which incorporates the work due to applied stress $\sigma$, can be defined as

$$
G(\varepsilon, \sigma, T)=\psi(\varepsilon, T)-\sigma \varepsilon,
$$

where $\sigma$ is the stress conjugate to $\varepsilon$. Given a stress $\sigma$ at temperature $T$, stable equilibrium strains must satisfy

$$
\frac{\partial G}{\partial \varepsilon}=0 \text { and } \frac{\partial^{2} G}{\partial \varepsilon^{2}}>0
$$

The equilibrium stress-strain relationship dictated by (13) yields a linear Hooke's law with isothermal stress-strain hysteresis, as illustrated in Figure 3. The strain values at which jumps occur coincide with the nodes $\pm \gamma_{M}(T)$ and $\pm \gamma_{A}(T)$ since they are also inflection points of the Helmholtz energy. We define the temperature-dependent transformation stresses

$$
\sigma_{A}(T)=E_{A} \gamma_{A}(T)
$$

and

$$
\sigma_{M}(T)=E_{M}\left(\gamma_{M}(T)-\varepsilon_{T}\right),
$$

corresponding to the stress values at the jumps.

For a fixed stress and varying temperatures, the necessary conditions in (13) yield a hysteretic strain-temperature hysteresis relation having the form illustrated in Figure 4. For the stress-free Helmholtz free energy, we specify $T_{M}$ and $T_{A}$ as temperatures where austenite and martensite, respectively, become unstable. In the case of the Gibbs free energy, the temperature values where austenite and martensite become unstable depend on stress and we denote 

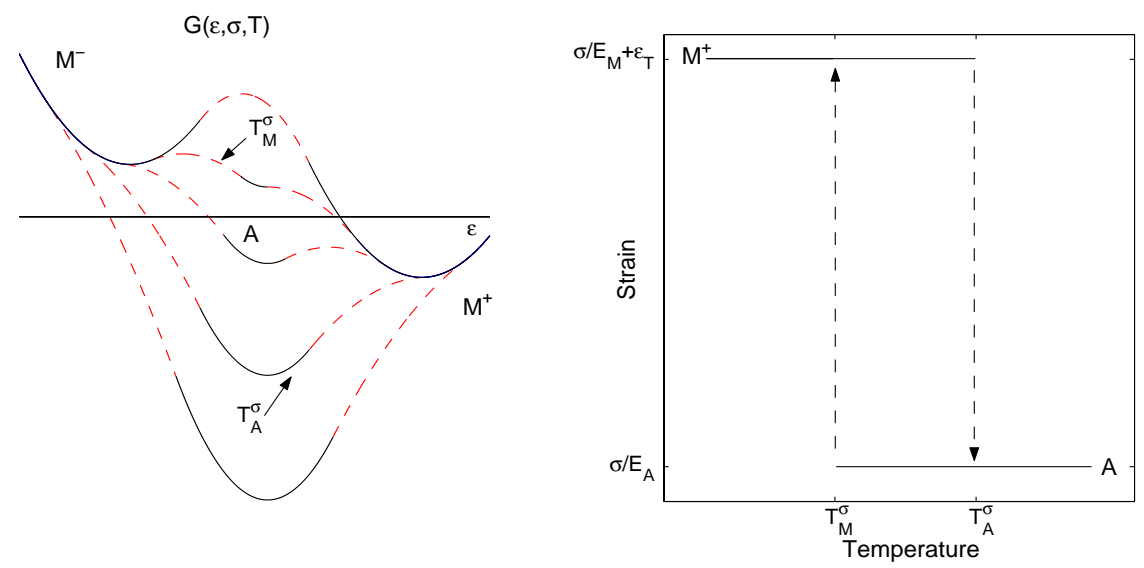

Fig. 4. The specific Gibbs free energy (12) at a fixed stress and varying temperatures. The equilibrium conditions (13) yield a hysteretic strain-temperature relation with jumps at the stress-dependent transformation temperatures $T_{M}^{\sigma}$ and $T_{A}^{\sigma}$.

them by $T_{M}^{\sigma}$ and $T_{A}^{\sigma}$. After describing the chemical free energy in the next section, we derive relations for $\sigma_{A}, \sigma_{M}, T_{M}^{\sigma}$, and $T_{A}^{\sigma}$.

\subsubsection{Chemical Free Energy}

In a manner similar to that employed in $[5,31,68,94,96]$, we model the chemical free energies as

$$
\begin{aligned}
\beta_{\alpha}(T) & =\int_{T_{R}}^{T} c_{\alpha} d T+u_{\alpha}-T s_{a} \\
& =c_{\alpha}\left(T-T_{R}\right)+u_{\alpha}-T s_{\alpha}
\end{aligned}
$$

where $c_{\alpha}$ are specific heat capacities, which we assume to be explicitly temperatureindependent. The constants $u_{\alpha}$ denote the internal energies at reference temperature $T_{R}$, and the $s_{\alpha}$ are specific entropies of the form

$$
\begin{aligned}
s_{\alpha} & =\int_{T_{R}}^{T} \frac{c_{\alpha}}{T} d T+\eta_{\alpha} \\
& =c_{\alpha} \ln \left(\frac{T}{T_{R}}\right)+\eta_{\alpha},
\end{aligned}
$$

where $\eta_{\alpha}$ are the constant entropies at $T_{R}$. The difference in the chemical potentials with a common reference temperature simplifies to

$$
\Delta \beta(T)=\Delta u-\Delta \eta T+\Delta c\left[T-T_{R}-T \ln \left(\frac{T}{T_{R}}\right)\right]
$$

where $\Delta u=u_{A}-u_{M}, \Delta \eta=\eta_{A}-\eta_{M}$, and $\Delta c=c_{A}-c_{M}$. As done in [41,68,71], we choose the reference temperature to coincide with the equilibrium temper- 
ature $T_{e q}$ where $\Delta \beta\left(T_{e q}\right)=0$. Therefore,

$$
\begin{aligned}
\Delta \beta\left(T_{R}\right) & =\Delta u-\Delta \eta T_{R} \\
& =\Delta u-\Delta \eta T_{e q}=0 .
\end{aligned}
$$

This then implies that, $T_{R}=T_{e q}=\Delta u / \Delta \eta$. We specify that $T_{e q}$ satisfies the conditions $T_{e q}>0$ and $T_{M}<T_{e q}<T_{A}$. Therefore, $\Delta u$ and $\Delta \eta$ are nonzero and do not differ in sign for $T_{R}=T_{e q}$.

We now show that $\Delta u, \Delta \eta>0$. We first note that

$$
\begin{aligned}
\frac{\partial \Delta \beta}{\partial T} & =-\Delta s \\
& =-\Delta c \ln \left(\frac{T}{T_{e q}}\right)-\Delta \eta
\end{aligned}
$$

Suppose $\Delta \eta<0$. If $\Delta c \leq 0$, then $\frac{\partial \Delta \beta}{\partial T}>0$ and $\Delta \beta(T)>0$ for all $T>T_{e q}$. However, as discussed in Section 2.1.1, we require $\Delta \beta\left(T_{A}\right)<0$. Hence, $\Delta \eta$ must be positive when $\Delta c \leq 0$.

If $\Delta c>0$, then $\frac{\partial \Delta \beta}{\partial T}>0$ and $\Delta \beta(T)<0$ for all positive $T<T_{e q}$. However, we require $\Delta \beta\left(T_{M}\right)>0$. So, $\Delta \eta$ must be positive when $\Delta c>0$. Therefore, we have $\Delta u>0$ and $\Delta \eta>0$.

The phase-dependent specific heat capacity of SMAs is not well-understood and measured values vary greatly. Both positive and negative values of $\Delta c$ have been measured $[26,41,69]$ and some SMA models assume that $\Delta c=0$ with little or no experimental evidence $[68,94]$. The assumption that $\Delta c=0$ is equivalent to making a first order Taylor approximation to (18) about $T=T_{R}$ since

$$
\Delta \beta(T)=\Delta u-\Delta \eta T-\frac{\Delta c T_{R}}{2 !}\left(T / T_{R}-1\right)^{2}+\mathcal{O}\left(\left(T / T_{R}-1\right)^{3}\right) .
$$

Of course, the first-order truncation is a good approximation in cases where temperatures vary only in a neighborhood of $T_{R}$ and when $\Delta c \approx 0$.

We can write (18) in the non-dimensional form

$$
b(\theta)=1-\theta+a[\theta-1-\theta \ln (\theta)],
$$

where $b(\theta)=\Delta \beta(T) / \Delta u, \theta=T / T_{e q}$, and $a=\Delta c / \Delta \eta$. Figure 5 illustrates (21) for $a \geq 0$.

As discussed in Section 2.1.1, for (18) to be consistent with (10) at temperatures below $T_{M}$, we require $\Delta \beta(T) \leq E_{M} \varepsilon_{T}^{2} / 2$. This condition can be satisfied for all $T$ only when $a \geq 0$. For $a>0, b(\theta)$ is concave with a single maximum 


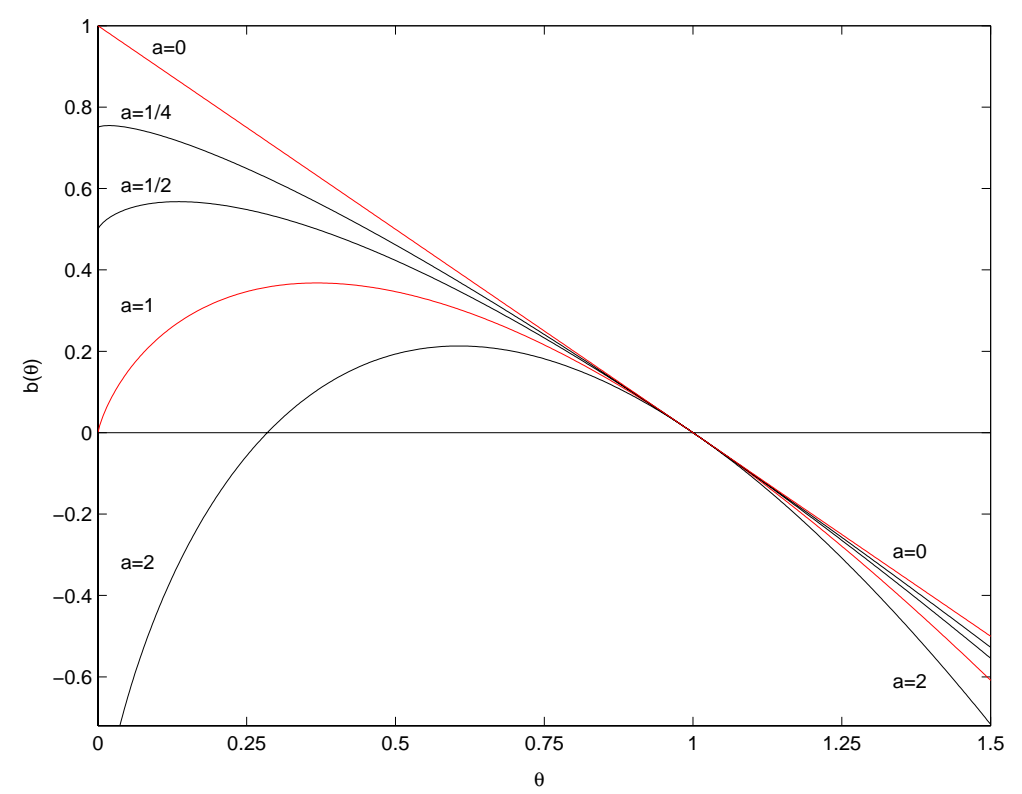

Fig. 5. The non-dimensional chemical free energy (21) for various $\Delta c \geq 0$ $(a=0,1 / 4,1 / 2,1,2)$. The general first-order approximation corresponds to the $\Delta c=0(a=0)$ case.

$b_{\max }$ at $\theta=e^{-1 / a}$. Therefore,

$$
b_{\max }=1+a\left(e^{-\frac{1}{a}}-1\right) \leq \frac{1}{2} \frac{E_{M} \varepsilon_{T}^{2}}{\Delta u} .
$$

Also, we note that while for all values of $a$ there exists a zero at $\theta=1$, for $a \geq 1$ there exists a second zero that does not correspond to a martensiteaustenite equilibrium temperature (see Figure 5). Therefore, combining (22) with $a<1$, we establish the condition

$$
e^{-1}<1-a\left(1-e^{-\frac{1}{a}}\right) \leq \frac{1}{2} \frac{E_{M} \varepsilon_{T}^{2}}{\Delta u}
$$

on the chemical free energy parameters to remain consistent with our definition of the Helmholtz free energy at low temperatures. If energy parameters are such that (23) is violated (e.g., measured $\Delta c<0$ ), we enforce consistency by setting $\Delta \beta(T)=E_{M} \varepsilon_{T}^{2} / 2$ for all $T<T_{M}$ where $\Delta \beta(T)>E_{M} \varepsilon_{T}^{2} / 2$ and setting $\Delta \beta(T)=0$ for all $T<T_{M}$ where $\Delta \beta(T)<0$.

\subsubsection{Local Transformation Stresses and Temperatures}

Having specified the chemical free energy $\Delta \beta(T)$ in (18), we wish to determine the explicit temperature-dependence of the local transformation stresses (14) and (15). Expressions for the transformation stresses in SMAs typically are determined from an approximation of a modified Clausius-Clapeyron equation, 
which describes the effect of hydrostatic pressure on transformation temperatures in a thermodynamic system $[1,41,71,82,103]$. However, we can obtain the expressions directly from (6) and (11).

For $T \geq T_{M}$, (6) is underdetermined given only $\Delta \beta(T)$. As an additional constraint we introduce

$$
\delta=\sigma_{A}(T)-\sigma_{M}(T)
$$

where $\delta$ is a material-dependent energy density that characterizes the hysteresis size [94]. In general, $\delta$ is temperature-dependent, as observed in $[41,71,95]$. In our present treatment, we approximate $\delta$ as a constant and we introduce an implicit temperature-dependence in Section 2.4.

It follows from the construction of the Gibbs potential that $\sigma_{A}(T)>\sigma_{M}(T)$ for $T \geq T_{M}$. Furthermore, $\gamma_{M}(T) \geq 0$ in (15) for all $T$ from which it follows that $\delta \in\left(0, E_{M} \varepsilon_{T}\right]$. With (24), we can formulate (6) solely in terms of $\sigma_{A}(T)$. This yields

where

$$
F\left(\sigma_{A}(T)\right)=\frac{\delta \varepsilon_{T}}{2}-\Delta \beta(T)
$$

$$
F(\sigma)=\sigma\left[\varepsilon_{T}+\frac{\left(1-E_{r}\right)}{2 E_{M}}(\sigma-\delta)\right],
$$

and $E_{r}=E_{M} / E_{A}$ is less than one for shape memory compounds. Solving (25) for $\sigma_{A}(T)$ and recalling that $\sigma_{A}(T) \geq 0$ yields

$$
\sigma_{A}(T)=\frac{\delta}{2}-\frac{E_{M}}{1-E_{r}} \varepsilon_{T}+\sqrt{\frac{\delta^{2}}{4}+\frac{E_{M}}{1-E_{r}}\left(\frac{E_{M}}{1-E_{r}} \varepsilon_{T}^{2}-2 \Delta \beta(T)\right)} .
$$

Note that the radicand in (27) is guaranteed to be non-negative provided

$$
\Delta \beta(T) \leq \frac{1}{1-E_{r}} \frac{E_{M} \varepsilon_{T}^{2}}{2}+\frac{1-E_{r}}{E_{M}} \frac{\delta^{2}}{8} .
$$

Since $\left(1-E_{r}\right)^{-1}>1$ and we enforce $\Delta \beta(T) \leq E_{M} \varepsilon_{T}^{2} / 2$, (28) always holds.

The expression for $\sigma_{M}(T)$, when $T \geq T_{M}$, follows from (24) and (27). For $T<T_{M},(11)$ yields

$$
\sigma_{M}(T)=-\frac{2}{\varepsilon_{T}} \Delta \beta(T) .
$$

In this case, $\sigma_{M}(T)$ corresponds to the critical detwinning stress to the $M^{-}$ variant.

We can also derive relations for the transformation temperatures. The stressfree transformation temperature $T_{M}$ satisfies

$$
\Delta \beta\left(T_{M}\right)=\frac{\delta \varepsilon_{T}}{2}
$$


since $\sigma_{A}\left(T_{M}\right)=0$. Furthermore, at $T_{A}$ where martensite becomes unstable, $\gamma_{M}\left(T_{A}\right)=\varepsilon_{T}$. Hence,

$$
\Delta \beta\left(T_{A}\right)=-\frac{\delta \varepsilon_{T}}{2}
$$

Under a nonzero stress $\sigma$, a transformation from austenite to martensite occurs when $\sigma_{A}\left(T_{M}^{\sigma}\right)=\sigma$. In addition, $\sigma_{M}\left(T_{A}^{\sigma}\right)=0$ holds. Therefore, the stressdependent transformation temperatures satisfy

$$
\Delta \beta\left(T_{M}^{\sigma}\right)=\frac{\delta \varepsilon_{T}}{2}-F(\sigma)
$$

and

$$
\Delta \beta\left(T_{A}^{\sigma}\right)=\frac{\delta \varepsilon_{T}}{2}-F(\sigma+\delta)
$$

whose solutions represent the inverses to $\sigma_{A}(T)$ and $\sigma_{M}(T)$, respectively. For $\Delta c \neq 0,(30)-(33)$ must be solved numerically.

Figure 6 illustrates the relationship between the transformation stresses and temperatures for $a=0,1$ and nominal values of the material constants. The temperature-dependent austenite-to-martensite critical stress $\sigma_{A}$ corresponds to the stress-dependent austenite-to-martensite transformation temperature $T_{M}^{\sigma}$. Similarly, the martensite-to-austenite unloading stress $\sigma_{M}$ for temperatures $T \geq T_{A}$ corresponds to $T_{A}^{\sigma}$. For $T<T_{A},-\sigma_{M}(T)$ is the critical detwinning stress. The plateau exhibited by the critical stress for low temperatures is a result of enforcing $\Delta \beta(T) \leq E_{M} \varepsilon_{T}^{2} / 2$ and corresponds to $\gamma_{M}(T)=0$.

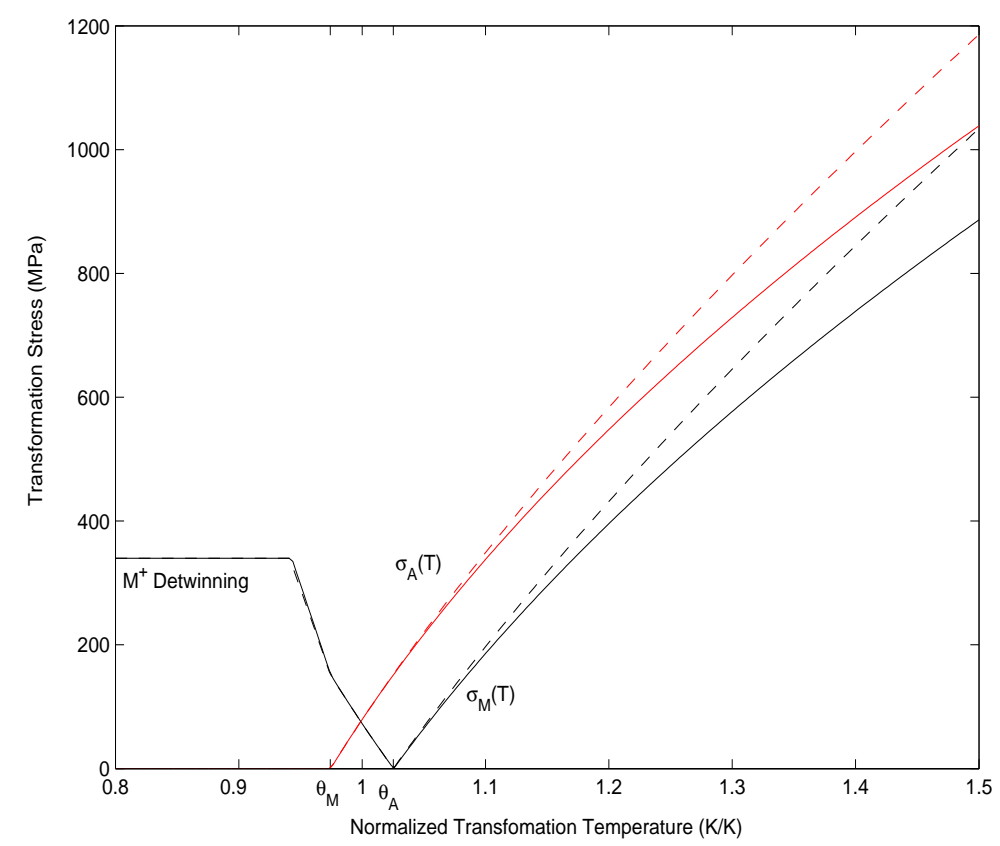

Fig. 6. The phase transformation stresses and transformation temperatures normalized by $T_{e q}$ with $\theta_{M}=T_{M} / T_{e q}$ and $\theta_{A}=T_{A} / T_{e q}$. The dashed lines are for $a=1$ and the solid lines are for $a=0$. 


\subsection{Phase Evolution}

Based on the local free energy, we follow the approach in [3,94] and model the phase evolution with the rate laws

$$
\begin{aligned}
& \dot{x}_{-}(t)=P_{A-} x_{A}(t)-P_{-} x_{-}(t) \\
& \dot{x}_{A}(t)=P_{-} x_{-}(t)-P_{A-} x_{A}(t)+P_{+} x_{+}(t)-P_{A+} x_{A}(t) \\
& \dot{x}_{+}(t)=P_{A+} x_{A}(t)-P_{+} x_{+}(t),
\end{aligned}
$$

where $\cdot$ denotes differentiation in time. The functions $P_{ \pm}=P_{ \pm}(\sigma, T)$ denote the transition rates for $M^{ \pm}$lattice elements (transforming to either austenite or a martensite variant), while $P_{A \pm}=P_{A \pm}(\sigma, T)$ denote the transition rates for austenite transforming to $M^{ \pm}$. Using the conservation relation (1), the rate law reduces to

$$
\begin{aligned}
& \dot{x}_{-}(t)=-\left(P_{-}+P_{A-}\right) x_{-}(t)-P_{A-} x_{+}(t)+P_{A-} \\
& \dot{x}_{+}(t)=-\left(P_{+}+P_{A+}\right) x_{+}(t)-P_{A+} x_{-}(t)+P_{A+} .
\end{aligned}
$$

For the case $T \leq T_{M}$, the rate law for martensite variants simplifies to a single ODE

$$
\dot{x}_{+}(t)=-\left(P_{+}+P_{-}\right) x_{+}(t)+P_{-},
$$

since $x_{-}(t)+x_{+}(t)=1$.

\subsection{Transition Rates}

We formulate the transition rates using the classical transition state theory of nonequilibrium processes $[36,86]$. The theory stipulates that, due to thermal excitation, lattice particles vibrate about equilibrium positions in energy wells. Furthermore, it contends that the vibrations are manifested as a temperaturedependent probability that the particles can overcome energy barriers separating the wells. For example, in the case of the local equilibrium conditions (13), the theory implies that thermally excited layers can transform from $A$ to $M^{+}$even when $\sigma_{A}$ and $T_{M}^{\sigma}$ are not reached.

Transition state theory quantifies the transition rate by combining the likelihood that a particle will overcome a barrier with the frequency of attempts a particle makes to overcome a barrier. The theory derives the likelihood that a lattice element will attain specific energy $G$ using the Boltzmann relation

$$
\mu(G)=C e^{-G V / k_{B} T}
$$

where $C$ is a normalization factor chosen to yield a likelihood of one when integrating $\mu(G)$ over all admissible energy states. The parameter $k_{B}=$ 
$1.380658 \times 10^{-23} \mathrm{~J} \mathrm{~K}^{-1}$ is the Boltzmann constant and we assume the representative layer volume $V$ is constant. We compute the transition rate for a martensite layer by multiplying (37) with the attempt frequency [86] to yield

$$
P_{ \pm}(\sigma, T)=\sqrt{\frac{k_{B} T}{2 \pi m V^{2 / 3}}} \frac{e^{-G\left( \pm \gamma_{M}, \sigma, T\right) V / k_{B} T}}{\int_{ \pm \gamma_{M}}^{ \pm \infty} e^{-G(\gamma, \sigma, T) V / k_{B} T} d \gamma}
$$

given a stress $\sigma$ and temperature $T$, where $m$ is the mass of the layer and $G\left( \pm \gamma_{M}, \sigma, T\right)$ is the Gibbs potential (12) at nodes $\pm \gamma_{M}(T)$. In transition state theory, $G\left( \pm \gamma_{M}, \sigma, T\right)$ is termed the activation energy. Note that we set the integration limits for the normalization factor to cover all possible stable (or metastable) martensite equilibrium states, neglecting the unstable states defined by the concave parabolae in (4). Likewise, the transition rate for an austenite layer $\left(T>T_{M}\right)$ is given by

$$
P_{A \pm}(\sigma, T)=\sqrt{\frac{k_{B} T}{2 \pi m V^{2 / 3}}} \frac{e^{-G\left( \pm \gamma_{A}, \sigma, T\right) V / k_{B} T}}{\int_{-\gamma_{A}}^{\gamma_{A}} e^{-G(\gamma, \sigma, T) V / k_{B} T} d \gamma} .
$$

Using (14), (15), and (25), we simplify the normalization integrals into transcendental functions of transformation stresses. This yields

$$
P_{ \pm}(\sigma, T)=\frac{1}{\tau} \frac{\sqrt{E_{r}}}{\operatorname{erfcx}\left[\left(\sigma_{M} \mp \sigma\right) / \sqrt{2} \omega_{M}\right]},
$$

where

$$
\operatorname{erfcx}(y)=e^{y^{2}}(1-\operatorname{erf}(y))
$$

is the scaled complementary error function and $\operatorname{erf}(y)=2 / \sqrt{\pi} \int_{0}^{y} e^{-r^{2}} d r$ is the standard error function. It then follows that $P_{A \pm}$ can be expressed as

$$
\begin{aligned}
P_{A \pm}(\sigma, T)=\frac{1}{\tau}\left\{ \pm \operatorname{erfcx}\left[\left(\sigma \mp \sigma_{A}\right) / \sqrt{2} \omega_{A}\right]\right. & \\
& \left.\mp e^{\mp 2 \sigma_{A} \sigma / \omega_{A}{ }^{2}} \operatorname{erfcx}\left[\left(\sigma \pm \sigma_{A}\right) / \sqrt{2} \omega_{A}\right]\right\}^{-1} .
\end{aligned}
$$

For $T \leq T_{M}$, where $\gamma_{A}(T)=0$, there is at most one energy state, so we define $P_{A \pm}(\sigma, T) \equiv \tau^{-1}$. The quantity

$$
\tau=\pi \sqrt{\frac{m}{E_{A} V^{1 / 3}}}
$$

represents a transformation relaxation time and the functions

$$
\omega_{\alpha}(T)=\sqrt{E_{\alpha} \frac{k_{B} T}{V}}
$$

quantify thermal energy densities for martensite and austenite. 
Asymptotic analysis in [4] shows that $\operatorname{erfcx}(x) \approx(\sqrt{\pi} x)^{-1}$ for large positive arguments and it is unbounded as $x \rightarrow-\infty$. Therefore, $\lim _{\sigma \rightarrow \pm \infty} P_{ \pm}(\sigma, T)=$ 0 and $\lim _{\sigma \rightarrow \pm \infty} P_{A \pm}(\sigma, T)=\infty$. Furthermore, at fixed temperatures

$$
\lim _{\omega_{M} \rightarrow 0} P_{ \pm}(\sigma, T)=\left\{\begin{array}{cr}
0 & \pm \sigma>\sigma_{M}(T) \\
\tau^{-1} \sqrt{E_{r}} & \pm \sigma=\sigma_{M}(T) \\
\infty & \pm \sigma<\sigma_{M}(T)
\end{array}\right.
$$

and

$$
\lim _{\omega_{A} \rightarrow 0} P_{A \pm}(\sigma, T)=\left\{\begin{array}{c}
0 \quad \pm \sigma<\sigma_{A}(T) \\
\tau^{-1} \pm \sigma=\sigma_{A}(T) \\
\infty \pm \sigma>\sigma_{A}(T)
\end{array}\right.
$$

The unbounded limits are a result of our mathematical description of the Boltzmann likelihoods. Physically, the unboundedness implies that a transformation will necessarily occur at precise thresholds of stress and temperature. Therefore, for small relative thermal energy (large volume $V$ and no relaxation), the transformation behavior is governed by the deterministic equilibrium conditions (13). Otherwise, the stochastic transition rates imply that transformations can occur even when (13) do not hold. Furthermore, $\lim _{\omega_{M} \rightarrow \infty} P_{ \pm}(\sigma, T)=\tau^{-1} \sqrt{E_{r}}$ (Boltzmann likelihood of one), which is the value for $\sigma= \pm \sigma_{M}(T)$, and $\lim _{\omega_{A} \rightarrow \infty} P_{A \pm}(\sigma, T)=\infty$. When implementing (40) and (42), we define maximum likelihoods to occur at the transformation stresses, which implies that

$$
P_{ \pm}(\sigma, T)=\left\{\begin{array}{cl}
\tau^{-1} \sqrt{E_{r}} & \pm \sigma \leq \sigma_{M}(T) \\
\frac{\tau^{-1} \sqrt{E_{r}}}{\operatorname{erfcx}\left[\left(\sigma_{M} \mp \sigma\right) / \sqrt{2} \omega_{M}\right]} & \pm \sigma>\sigma_{M}(T) .
\end{array}\right.
$$

Likewise, for $T \geq T_{M}$ and $|\sigma|<\sigma_{A}$, (42) holds. Otherwise, we have that

$$
P_{A \pm}(\sigma, T)=\left\{\begin{array}{cc}
\tau^{-1} & T<T_{M} \\
\frac{\tau^{-1}}{\operatorname{erf}\left[\sqrt{2} \sigma_{A} / \omega_{A}\right]} \pm \sigma \geq \sigma_{A}(T) .
\end{array}\right.
$$

Note that (48) is unbounded, since

$$
\lim _{T \rightarrow T_{M}+} P_{A \pm}\left( \pm \sigma_{A}, T\right)=\infty
$$

For this case, we heuristically assign a maximum greater than or equal to $\tau^{-1}$. Figure 7 illustrates (47) and (48) for small thermal energy in stresstemperature space. 


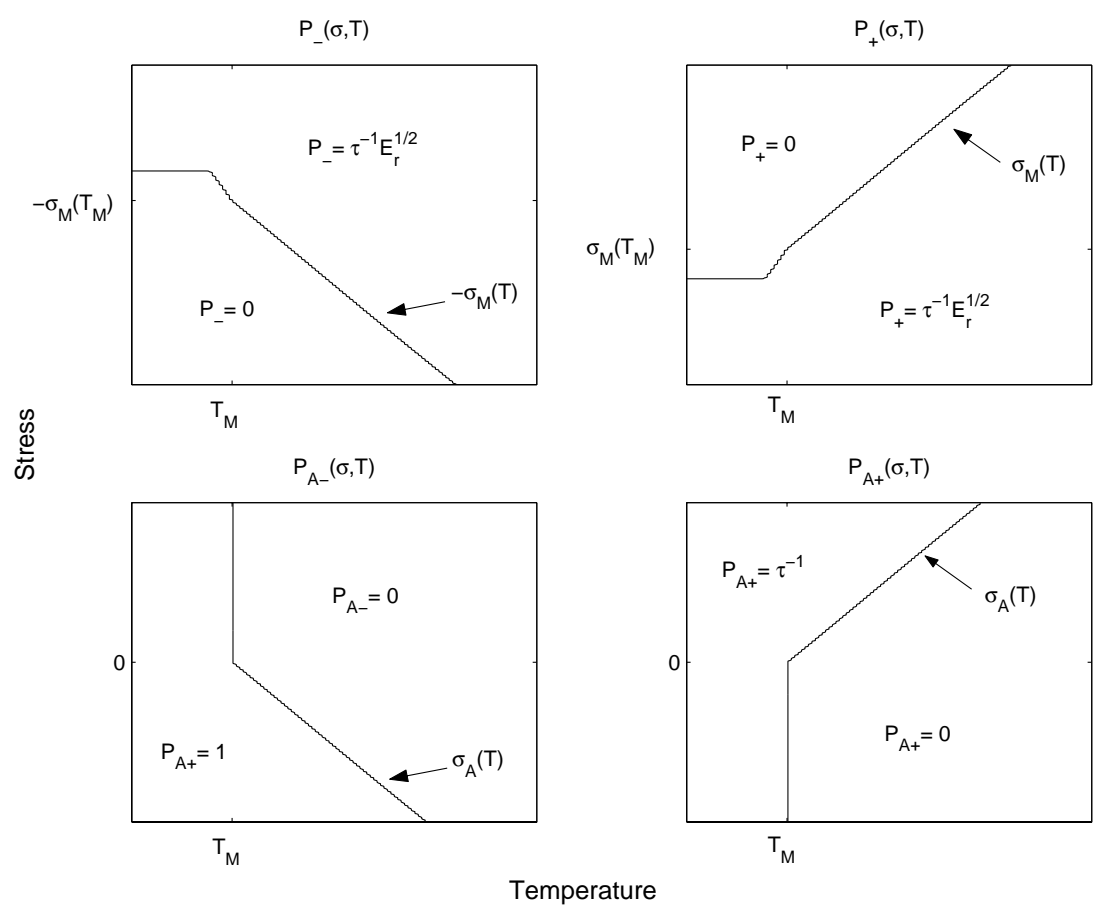

Fig. 7. The transition rates $P_{ \pm}$and $P_{A \pm}$ for varying temperature and stress at low thermal energy. The solid lines mark the transformation boundaries defined by transformation stresses and temperatures.

Ultimately, both (40) and (42) have qualities similar to those of a normal distribution about $\sigma$, where the transformation stresses $\sigma_{\alpha}$ act as means and $\omega_{\alpha}$ as standard deviations. Increasing thermal energy densities (large variances) increases the likelihood that transformations will occur when (13) do not hold. Diminishing thermal energy densities (small variances) restrict transformations to occur only when (13) are satisfied. Figure 8 illustrates the transition rates for increasing $\omega_{\alpha}$ at a fixed temperature and decreasing activation volumes.

\subsection{Expected Local Strains}

Given the temperature evolution $T(t)$ and the phase fractions modeled by (35), we can now quantify the thermomechanical response of the material. Boltzmann statistics govern the response of individual martensite and austenite layers. Accounting for thermal energy, the expected strain exhibited by martensite layers are given by

$$
\left\langle\varepsilon_{-}\right\rangle=\int_{-\infty}^{-\gamma_{M}} \gamma \mu(G(\gamma, \sigma, T)) d \gamma
$$



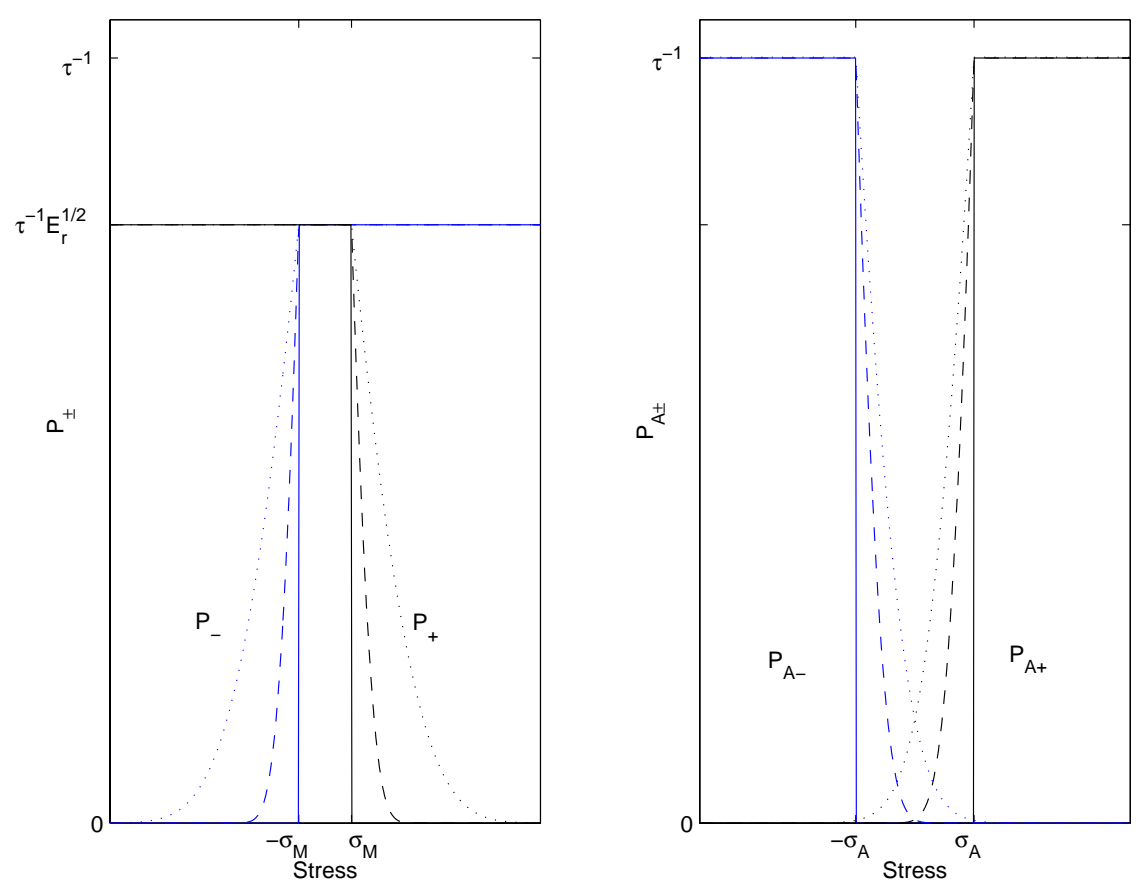

Fig. 8. The transition rates $P_{ \pm}$and $P_{A \pm}$ for a fixed temperature $T>T_{A}$ and varying stress. The solid, dashed, and dotted lines are for $k_{B} / V=0,10^{2}$, and $10^{3}$, respectively. The function values are fixed once a transformation stress is reached.

and

$$
\left\langle\varepsilon_{+}\right\rangle=\int_{\gamma_{M}}^{\infty} \gamma \mu(G(\gamma, \sigma, T)) d \gamma
$$

where the Boltzmann likelihood $\mu(G)$ from $(37)$ acts as a probability density function. Likewise for austenite layers $\left(T>T_{M}\right)$, the expected strains are

$$
\left\langle\varepsilon_{A}\right\rangle=\int_{-\gamma_{A}}^{\gamma_{A}} \gamma \mu(G(\gamma, \sigma, T)) d \gamma
$$

For $T \leq T_{M}, \mu(G)=1$ and $\gamma_{A}=0$, so $\left\langle\varepsilon_{A}\right\rangle=0$. Simplifying the integrals yields

$$
\left\langle\varepsilon_{ \pm}\right\rangle= \pm \sqrt{\frac{2}{\pi}} \frac{\tau \omega_{M}(T)}{E_{M} \sqrt{E_{r}}} P_{ \pm}(\sigma, T)+\left(\frac{\sigma}{E_{M}} \pm \varepsilon_{T}\right)
$$

and

$$
\left\langle\varepsilon_{A}\right\rangle=\sqrt{\frac{2}{\pi}} \frac{\tau \omega_{A}(T)}{E_{A}}\left[P_{A-}(\sigma, T)-P_{A+}(\sigma, T)\right]+\frac{\sigma}{E_{A}}
$$

in terms of the transition rates (40) and (42). From (53) and (54), it is apparent that the expected strains are solutions to (13) with perturbations whose magnitudes depend on the thermal energy. Note that at fixed temperatures

$$
\lim _{\omega_{M} \rightarrow 0}\left\langle\varepsilon_{ \pm}\right\rangle=\left\{\begin{array}{c}
\frac{\sigma}{E_{M}} \pm \varepsilon_{T} \pm \sigma \geq \sigma_{M}(T) \\
\pm \gamma_{M}(T) \pm \sigma<\sigma_{M}(T)
\end{array}\right.
$$



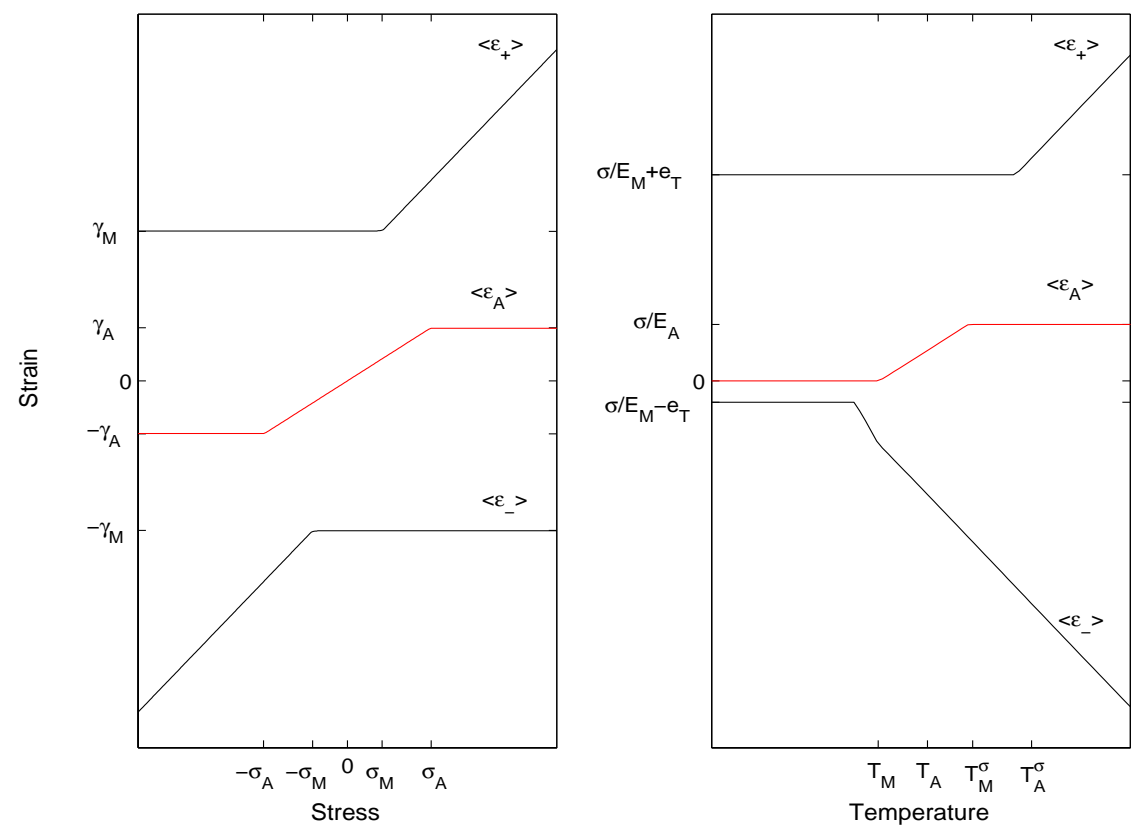

Fig. 9. The expectation strains (53) and (54) as a function of stress $\left(T>T_{A}\right)$ and temperature for low thermal energy.

and

$$
\lim _{\substack{\omega A \rightarrow 0 \\
T>T_{M}}}\left\langle\varepsilon_{A}\right\rangle=\left\{\begin{array}{cc}
\frac{\sigma}{E_{A}} & |\sigma| \leq \sigma_{A}(T) \\
\pm \gamma_{A}(T) \pm \sigma>\sigma_{A}(T)
\end{array}\right.
$$

Furthermore, $\lim _{\sigma \rightarrow \mp \infty}\left\langle\varepsilon_{ \pm}\right\rangle= \pm \gamma_{M}$ and $\lim _{\sigma \rightarrow \pm \infty}\left\langle\varepsilon_{A}\right\rangle= \pm \gamma_{A}$. Figure 9 illustrates the stress and temperature-dependence of (53) and (54).

With the expected strain of a layer in each phase thus formulated, the average local strain can be expressed as the weighted sum

$$
\bar{\varepsilon}_{m e c h}=\sum_{\alpha} x_{\alpha}\left\langle\varepsilon_{\alpha}\right\rangle .
$$

In addition to the strain $\varepsilon_{\text {mech }}$ attributed to mechanical deformation and phase transformation processes, there are strains due to local thermal expansion as the temperature varies over time. We model the local thermal strain as

$$
\bar{\varepsilon}_{\text {ther }}=\bar{\lambda}\left(x_{\alpha}\right)\left(T-T_{0}\right)
$$

for the phase-dependent thermal expansion coefficient $\bar{\lambda}\left(x_{\alpha}\right)=\sum_{\alpha} \lambda_{\alpha} x_{\alpha}(t)$, and initial temperature $T_{0}$. Therefore, the total average strain of a layer in response to a combination of applied stress and temperature is

$$
\bar{\varepsilon}=\bar{\varepsilon}_{\text {mech }}+\bar{\varepsilon}_{\text {ther }} .
$$

Typically, $\bar{\varepsilon}_{\text {ther }}$ is negligible compared to $\bar{\varepsilon}_{\text {mech }}$ for moderate temperature changes as would be encountered in bulk superelastic applications. How- 

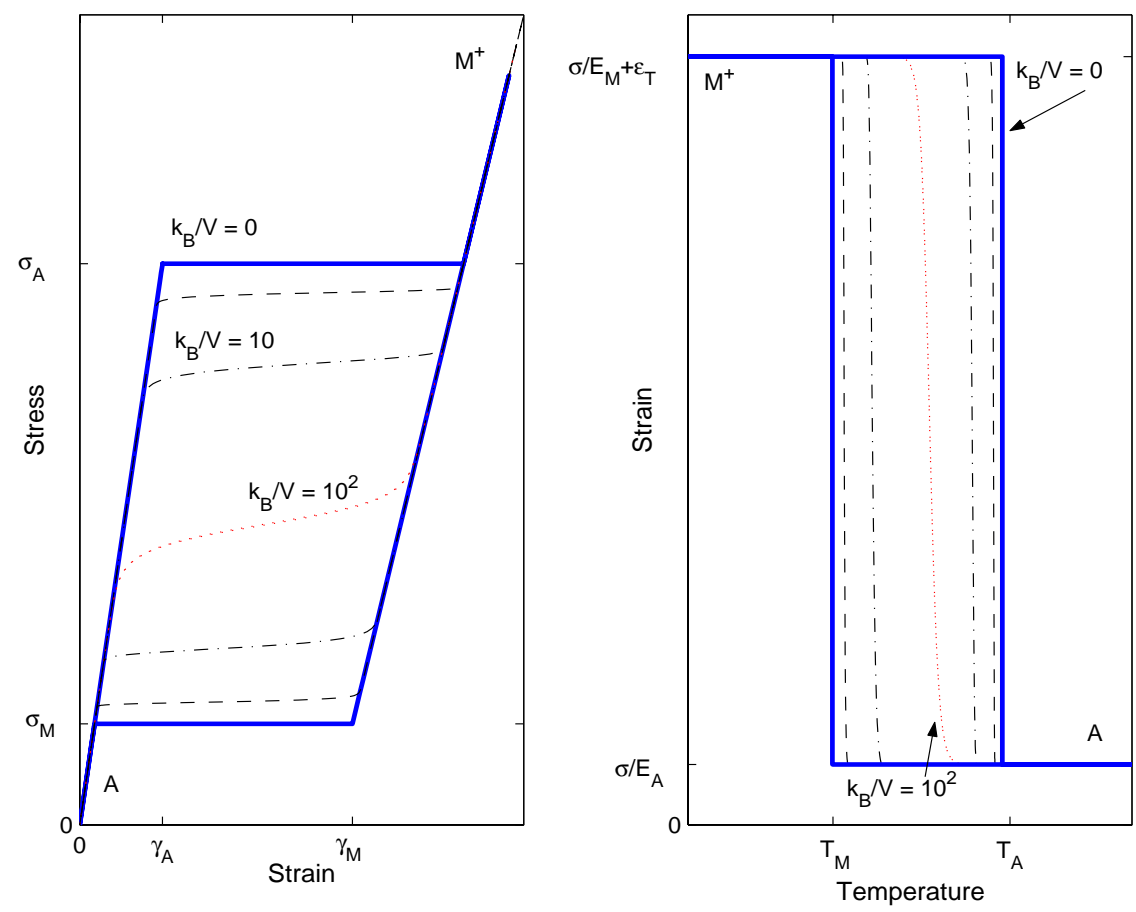

Fig. 10. Superelasticity (fixed $T>T_{A}$ ) and shape memory effect (fixed stress) hysteresis determined from (57). The largest hysteresis loops occur for $k_{B} / V=0$ and hysteresis diminishes to zero for increasing $k_{B} / V$.

ever for thin films in MEMS applications, thermal strains can have significant effects on microdevice behavior (e.g., see $[49,79]$ ).

Figure 10 illustrates stress-strain (superelastic) and temperature-strain (SME) hysteresis predicted by (57). For diminishing thermal energy, hysteresis increases to a maximum and corresponds to the equilibrium solution (13). For increasing thermal energy densities, more lattice layers overcome energy barriers before transformation thresholds are reached, resulting in a smaller hysteresis loops. If the thermal energy is sufficiently large, all energy barriers become surpassable at any stress and temperature. In this case, layers attain the global minimum of (12) and there is no hysteresis. We note that the diminishing hysteresis reflects an implicit temperature-dependence of $\delta$ in (24), where $\frac{\partial \delta}{\partial T}<0$ provided $\lim _{T \rightarrow \infty} \sigma_{\alpha}(T) / \omega_{\alpha}(T)=0$.

In the next section, we model the variation of temperature over time due to phase transformations and ambient conditions.

\subsection{Thermal Evolution}

The internal temperature of an SMA is coupled to the thermomechanical phase transformations and it strongly depends on operating conditions. We describe temperature changes in the material via a simplified balance of internal energy, 
similar to the developments in $[14,26,94]$. In this case,

$$
\bar{c}\left(x_{\alpha}\right) \dot{T}(t)=-\sum_{\alpha} h_{\alpha} \dot{x}_{\alpha}+H\left(x_{\alpha}, T\right),
$$

where $\bar{c}\left(x_{\alpha}\right)=\sum_{\alpha} c_{\alpha} x_{\alpha}(t)$ represents a linear mixture of phase-dependent specific heats. In the following subsections, we describe the components forming the right-hand-side of (60), which include transformation-induced heat generation and heat exchange with the environment.

\subsubsection{Transformation Enthalpy}

The first term on the right-hand side of (60) accounts for rate-dependent heat generation and absorption during phase transformations. The release and absorption of transformation enthalpies can be significant and is a source of the material self-heating observed in [95]. The specific enthalpies $h_{\alpha}$ have the form

$$
h_{\alpha}=g_{\alpha}+T s_{\alpha}
$$

In (61), $g_{\alpha}$ is a local minimum of (12) and $s_{\alpha}$ is the specific entropy from (17). Given stress $\sigma$ at temperature $T$,

$$
\begin{aligned}
& g_{A}=\frac{-\sigma^{2}}{2 E_{A}}+\Delta \beta(T) \\
& g_{ \pm}=\frac{-\sigma^{2}}{2 E_{M}} \mp \sigma \varepsilon_{T} .
\end{aligned}
$$

Since $\sum_{\alpha} \dot{x}_{\alpha}(t) \equiv 0$, we have

$$
\sum_{\alpha} h_{\alpha} \dot{x}_{\alpha}=-\left(h_{A}-h_{-}\right) \dot{x}_{-}-\left(h_{A}-h_{+}\right) \dot{x}_{+},
$$

and

$$
h_{A}-h_{ \pm}=\frac{\sigma^{2}}{2}\left(\frac{1}{E_{M}}-\frac{1}{E_{A}}\right) \pm \sigma \varepsilon_{T}+\Delta c\left(T-T_{R}\right)+\Delta u .
$$

The differences in martensite and austenite specific enthalpies in (65) are referred to as latent heats of transformation. Note that for $T \leq T_{M}$, (64) reduces to

$$
\sum_{\alpha} h_{\alpha} \dot{x}_{\alpha}=-2 \sigma \varepsilon_{T} \dot{x}_{+}
$$

\subsubsection{Heat Transfer}

Heat transfer is a phenomenon pertaining to a physical system of which an SMA is a component, rather than a constitutive property of the material. However, for basic actuator simulations and model comparisons to thermomechanical tests, we introduce the thermal interaction of the material with 
its environment via $H\left(x_{\alpha}, T\right)$ in equation (60). For our uniaxial model, we assume that the temperature is spatially uniform in SMAs such as thin films and wires. A uniform longitudinal temperature distribution can be realized in a thermal chamber or in transducers with resistive heating. Additionally we assume that internal temperature can be approximated effectively by models of heat transfer at the surface. We establish the validity of this assumption by calculating the dimensionless Biot number $B i$ (see $[27,47,69,96])$ given by

$$
B i=\frac{h_{c}}{k_{\alpha} \Omega}
$$

where $h_{c}$ is the phenomenological average convection heat transfer coefficient, $k_{\alpha}$ is the phase-dependent thermal conductivity, and $\Omega$ is the surface areato-volume ratio. For $B i \ll 1$, the temperature changes within the material are negligible relative to temperature differences between the surface and surroundings [47]. For SMAs such as NiTi, the conductivity $k_{\alpha}$ is on the order of $10 \mathrm{Wm}^{-1} \mathrm{~K}^{-1}$ and $h_{c}$ is on the order of 1 and $10^{2} \mathrm{Wm}^{-2} \mathrm{~K}^{-1}$, depending on ambient flow conditions [14,69]. Furthermore, for rectangular geometries of thickness $d$, width $w$ and length $L$,

$$
\Omega=2\left(\frac{1}{d}+\frac{1}{w}+\frac{1}{L}\right) .
$$

Likewise, for cylindrical geometries of diameter $d$,

$$
\Omega=2\left(\frac{2}{d}+\frac{1}{L}\right)
$$

Hence, for thin films and wires where $d \ll\{L, w\}, \Omega$ is $\mathcal{O}\left(d^{-1}\right)$ and $B i$ is $\mathcal{O}\left(10 \mathrm{~m}^{-1} d\right)$. In the applications under consideration, SMA thicknesses are at most $1 \mathrm{~mm}$, so $B i \ll 1$ is satisfied.

Incorporating surface convection and radiation, and resistive Joule heating, the term $H$ in (60) can be expressed as

$$
H\left(x_{\alpha}, T\right)=-\left(h_{c}+h_{r}\right) \Omega\left[T(t)-T_{E}(t)\right]+J\left(x_{\alpha}, T\right),
$$

where $T_{E}(t)$ is the prescribed external temperature. The radiation heat transfer coefficient is given by

$$
h_{r}=\epsilon \sigma^{B}\left(T^{2}+T_{E}^{2}\right)\left(T+T_{E}\right),
$$

where $\epsilon \in[0,1]$ is the surface emissivity $(\lesssim 0.50$ for metals, $\lesssim 0.20$ for films $)$ and $\sigma^{B}=5.67051 \times 10^{-8} \mathrm{Wm}^{-2} \mathrm{~K}^{-4}$ is the Stefan-Boltzmann constant. In general, $h_{c}$ is also (weakly) temperature-dependent and is estimated from empirical arguments involving surface geometry and external fluid flow. In our treatment, we assume $h_{c}$ is constant and we refer the reader to $[14,27,47,55,69,85,87]$ for theoretical and empirical aspects of $h_{c}$ pertinent to SMAs. Note that in 
forced convection conditions, $h_{c}$ is typically one to three orders of magnitude greater than $h_{r}$ over all temperatures.

Lastly, in $(70), J\left(x_{\alpha}, T\right)$ incorporates heat generation via resistive Joule heating. For current-controlled applications,

$$
J\left(x_{\alpha}, T\right)=\bar{\rho}^{e}\left(x_{\alpha}, T\right) \frac{I^{2}}{\zeta^{2}},
$$

where $I$ is the applied current and $\zeta$ is the cross sectional area of the current path. The effective resistivity

$$
\bar{\rho}^{e}\left(x_{\alpha}, T\right)=\sum_{\alpha}\left(\rho_{\alpha}^{e}+\lambda_{\alpha}^{e} T\right) x_{\alpha}(t)
$$

includes the phase-dependent electrical resistivities $\rho_{\alpha}^{e}$, and linear temperature coefficients of resistivity $\lambda_{\alpha}^{e}$. If Joule heating results from a prescribed potential difference, we have

$$
J\left(x_{\alpha}, T\right)=\frac{1}{\bar{\rho}^{e}\left(x_{\alpha}, T\right)} \frac{V_{e}^{2}}{L^{2}}
$$

where $V_{e}$ is the applied voltage and $L$ is the actuator length.

The temperature-dependence of resistivity can be significant when quantifying resistivity-temperature hysteresis $[106,111]$. However, in cases where Joule heating is an impulse heat source, as is typical for MEMS applications, the explicit temperature-dependence is negligible.

In general, the geometric quantities $\Omega, \zeta$, and $L$ are functions of uniaxial strain. For example, in a rectangular geometry $\Omega$ changes by a factor of $(1-\nu \varepsilon)^{-1}$, $\zeta$ by a factor of $(1-\nu \varepsilon)^{2}$, and $L$ by a factor of $(1+\varepsilon)$, where the isotropic Poisson ratio $\nu \in[0,0.5](\nu \approx 0.33$ for isochoric NiTi). Since typical actuator strains are at most on the order of $10 \%$, the relation (70) can change on the order of $5-15 \%$ due to deformations. To facilitate model computation, we approximate $\Omega, \zeta$, and $L$ as constants.

We have modeled the phase fractions, expected strains, and temperature with free energy expressions that are valid for local lattice behavior. Equation (59) also quantifies macroscopic strains for specially-prepared homogeneous, single-crystal SMAs in which the bulk lattice exhibits uniform local behavior. In the next section, we extend our uniform lattice model to more general inhomogeneous compounds, where the local thermomechanical behavior can vary throughout the material. 


\section{Nonuniform Lattice Model}

Typical single-crystal and polycrystalline SMA compounds contain precipitates, dislocations, and process-induced impurities. Moreover, inhomogeneous and polycrystalline materials have a nonuniform lattice structure that induces variations in the local material response. In this section, we model the hysteresis induced in nonuniform SMA lattices and the effects of nonuniform stress fields by employing a statistical homogenization technique. For a discussion of general statistical homogenization concepts, we refer the reader to $[62,86]$. Our approach is based on the assumption that measurements of macroscopic phenomena, such as stress-strain hysteresis and transformation temperatures, yield quantities that reflect a distribution of local variations. In the context of statistical homogenization, the uniform lattice model (59) predicts the expected local response when given effective macroscopic material parameters as input.

\subsection{Variations in Hysteresis}

One approach to account for inhomogeneities is to treat measurements of macroscopic hysteresis parameters, such as $\varepsilon_{T}$ and $\delta$, as manifestations of stochastic distributions, rather than deterministic values. In particular, variations of $\delta$ and $\varepsilon_{T}$ produce variations in the transformation thresholds $\sigma_{A}, \sigma_{M}$, $T_{M}$ and $T_{A}$ via (27), (30), and (31), and they reflect variations in the chemical free energy parameters. We model the effects of varying $\delta$ on the observed macroscopic strain $\varepsilon$ by considering the statistical average

$$
\varepsilon(\sigma, T)=\int_{0}^{\infty} \bar{\varepsilon}(\sigma, T ; \delta) p_{1}(\delta) d \delta,
$$

where $\bar{\varepsilon}$ is the local strain in (59), and $p_{1}$ is a probability density function defined on $[0, \infty)$. Reflecting the boundedness of $\delta$, we represent $p_{1}$ as a $\log$ normal distribution on the interval $\left(0, E_{M} \varepsilon_{T}\right]$, given $E_{M}$ and $\varepsilon_{T}$.

As detailed in [72], the normalized log-normal probability density function is given by

$$
p_{1}(\delta)=\frac{1}{\hat{z} \sqrt{2 \pi} \delta} e^{-\ln (\delta / \hat{\mu})^{2} / 2 \hat{z}^{2}}
$$

with parameters $\hat{z}>0$ and $\hat{\mu}>0$. As illustrated in Figure 11, the lognormal distribution is continuous and right-skewed, and the logarithm of the distributed variable has a normal distribution. Increasing $\hat{z}$ and $\hat{\mu}$ correspond to increasing skewness. In cases where mean $\bar{\delta}$ and standard deviation $z_{\delta}$ of $\delta$ are given (e.g., experimental estimates), the log-normal parameters can be computed via the relations

$$
\hat{\mu}=\bar{\delta}\left(\frac{z_{\delta}^{2}}{\bar{\delta}^{2}}+1\right)^{-\frac{1}{2}}, \quad \hat{z}^{2}=\ln \left(\frac{z_{\delta}^{2}}{\bar{\delta}^{2}}+1\right) .
$$




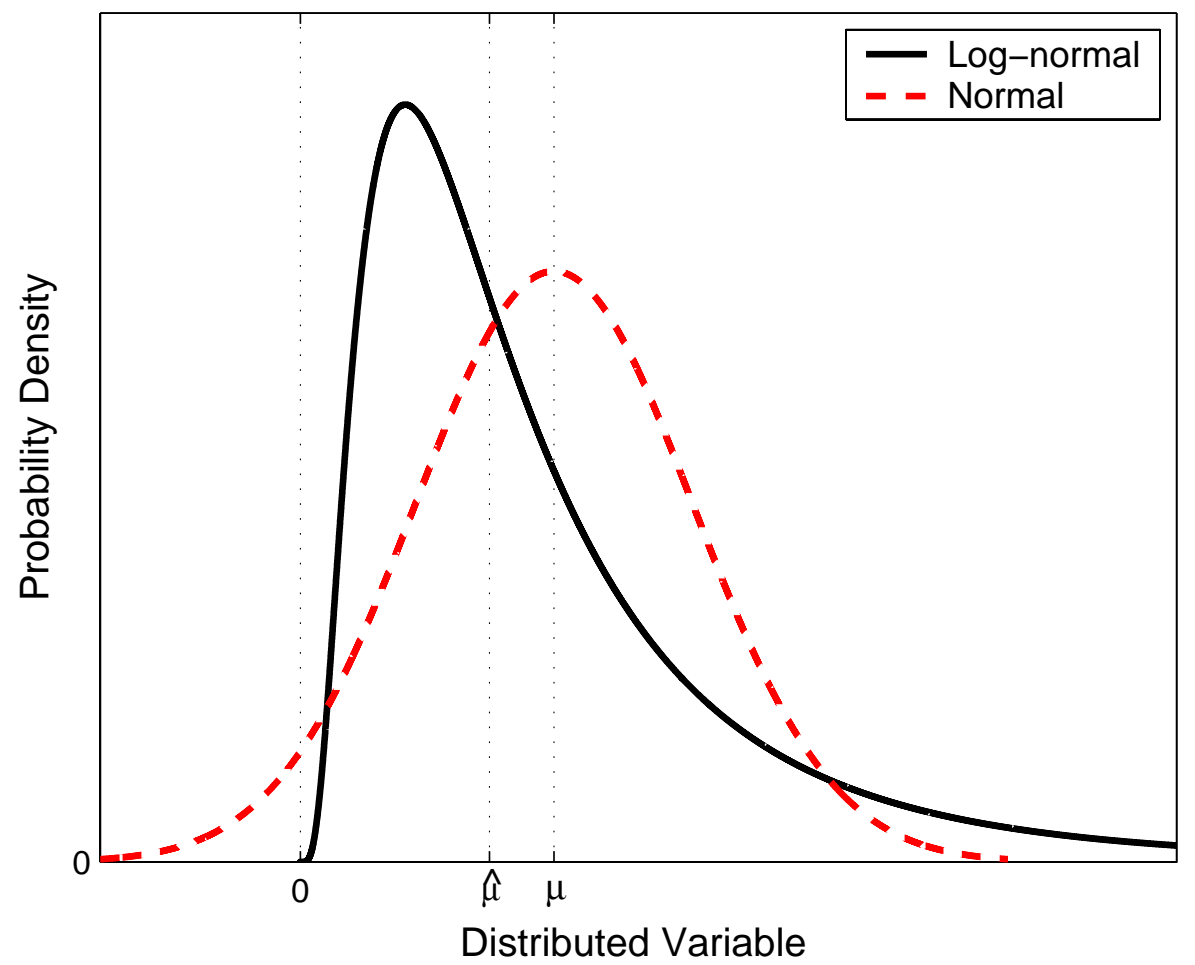

Fig. 11. The log-normal probability density function compared with its normal counterpart. The peak of the skewed log-normal function corresponds with the mode and the peak of the normal curve corresponds with the mean $\mu$. The areas under the curves correspond to the same probability.

\subsection{Effective Stress}

In addition to the model (74) which incorporates material inhomogeneities, we quantify the effects of nonuniform stress fields. We represent the local effective stress $\sigma_{e}$ as a perturbation of the macroscopic applied stress

$$
\sigma_{e}(t)=\sigma(t)+s,
$$

where $\sigma$ is the time-varying applied stress and $s$ is a constant perturbation, which we assume to be a random variable. The observed macroscopic strain is then a response to a distribution of effective stresses in the material

$$
\varepsilon(\sigma, T)=\int_{-\infty}^{\infty} \bar{\varepsilon}\left(\sigma_{e}, T\right) p_{2}(s) d s,
$$

where $p_{2}$ is a probability density function defined on $(-\infty, \infty)$. We assume $s$ is normally distributed, so we take $p_{2}$ to correspond with the normal distribution

$$
p_{2}(s)=\frac{1}{\sqrt{2 \pi} z_{\sigma}} e^{-s^{2} / 2 z_{\sigma}^{2}}
$$


with standard deviation $z_{\sigma}$. For certain geometries and load conditions, one can alternatively employ the trigonometric averaging procedure proposed in [83] to accommodate stress variations on rotated polycrystal grains. In particular, by distributing about the applied stress, we account for polycrystal grains undergoing stress and temperature-induced transformations at varying applied stresses and temperatures.

\subsection{Macroscopic Model}

The combination of (74) and (78) yields the relation

$$
\varepsilon(\sigma, T)=\int_{0}^{\infty} \int_{-\infty}^{\infty} \bar{\varepsilon}\left(\sigma_{e}, T ; \delta\right) p_{1}(\delta) p_{2}(s) d s d \delta
$$

for the total macroscopic response to a time-varying stress $\sigma(t)$ and an evolving internal temperature $T(t)$. In addition to the measured strain, the macroscopic phase fractions $x_{\alpha}$ and internal temperature $T$ are also representations of the underlying distributions. We compute these macroscopic quantities by replacing $\bar{\varepsilon}$ with $x_{\alpha}$ and $T$ in (80). Table 1 summarizes the parameters used in the local and macroscopic models. In the next section, we discuss implementation issues associated with the macroscopic model (80).

\section{Numerical Implementation and Simulations}

\subsection{Implementation}

In this section, we summarize aspects of the numerical implementation of the macroscopic model. To obtain the macroscopic strain response given a timevarying stress, environment temperature, and current or voltage, we solve the local model over distributed $\delta$ and $\sigma_{e}$ in (80). At the core of the local model is the nonlinear system of ODEs for the phase fractions and temperature. We express the system as

$$
\left[\begin{array}{c}
\dot{x}_{-}(t) \\
\dot{x}_{+}(t) \\
\dot{T}(t)
\end{array}\right]=\mathbf{M}\left(x_{\alpha}, T\right)\left[\begin{array}{c}
x_{-}(t) \\
x_{+}(t) \\
T(t)
\end{array}\right]+\left[\begin{array}{c}
P_{A-}(\sigma, T) \\
P_{A+}(\sigma, T) \\
H\left(x_{\alpha}, T\right)
\end{array}\right],
$$

where the state-dependent matrix $\mathbf{M}$ is given by

$$
\mathbf{M}\left(x_{\alpha}, T\right)=\left[\begin{array}{ccc}
1 & 0 & 0 \\
0 & 1 & 0 \\
\frac{L_{A-}}{\bar{c}\left(x_{\alpha}\right)} & \frac{L_{A+}}{\bar{c}\left(x_{\alpha}\right)} & \frac{1}{\bar{c}\left(x_{\alpha}\right)}
\end{array}\right]\left[\begin{array}{ccc}
-\left(P_{-}+P_{A-}\right) & -P_{A-} & 0 \\
-P_{A+} & -\left(P_{+}+P_{A+}\right) & 0 \\
0 & 0 & 1
\end{array}\right]
$$

and $L_{A \pm}=h_{A}-h_{ \pm}$. Recall that the latent heats and the transition rates depend on stress and temperature. The first step in solving the local model is 


\begin{tabular}{|c|c|}
\hline Parameter & Physical Description \\
\hline \multicolumn{2}{|r|}{ Physical Parameters } \\
\hline$E_{A}, E_{M}$ & Elastic moduli (linear). \\
\hline$\lambda_{A}, \lambda_{M}$ & Thermal expansion coefficients (linear). \\
\hline$c_{A}, c_{M}$ & Volumetric specific heat capacities $\left(\rho c_{V}\right)$. \\
\hline$\Delta u$ & Specific internal energy difference at reference. \\
\hline$\Delta \eta$ & Specific entropy difference at reference. \\
\hline$V$ & Activation volume (local). \\
\hline$\tau$ & Transformation relaxation time. \\
\hline \multicolumn{2}{|r|}{ Hysteresis Parameters } \\
\hline$\varepsilon_{T}$ & Transformation strain. \\
\hline $\bar{\delta}, z_{\delta}$ & Mean hysteresis thickness and standard deviation. \\
\hline$z_{\sigma}$ & Effective stress standard deviation. \\
\hline \multicolumn{2}{|r|}{ Heat Transfer Parameters } \\
\hline$\rho_{A}^{e}, \rho_{M}^{e}$ & Resistivities. \\
\hline$\lambda_{A}^{e}, \lambda_{M}^{e}$ & Temperature coefficients of resistivity. \\
\hline$\epsilon$ & Surface emissivity. \\
\hline$h_{c}$ & Convective heat transfer coefficient. \\
\hline \multicolumn{2}{|r|}{ Geometric Parameters } \\
\hline$L, d, w$ & Length, thickness (or diameter), width. \\
\hline
\end{tabular}

Table 1. Local and macroscopic model parameters.

to obtain initial data $x_{ \pm}(0)$ and $T(0)$ from experimental conditions. Typical initial conditions are the high-temperature state where $x_{ \pm}(0)=0$ or the unstressed low temperature (self-accommodating) state where $x_{ \pm}(0)=1 / 2$. Secondly, we calculate necessary model parameter estimates, as described in the next section, from given material data, such as the distributed value of $\delta$. Next, we solve (81) using the Matlab ODE routine ode15s, which is a variable order solver based on the numerical differentiation formulas. To compute the integrals in the transition rates and the expectation strains, we utilize the Matlab routines erf and erfcx, which approximate the functions directly with polynomials. Finally, we compute the thermal strains and total local strain given the expectation strains and the ODE solution.

To integrate the macroscopic model, we approximate the distribution integrals with composite Gauss-Legendre quadrature on finite intervals. Here we have adapted the scalar, univariate formulation described in [61] to the case of vector-valued functions. For scalar argument $x$ we define the vector-valued function $\vec{f}(x) \in \Re^{1 \times m}$. The $n$-point composite Gauss quadrature approxi- 
mates the component-wise integral $\vec{F} \in \Re^{1 \times m}$ on interval $\left[a_{x}, b_{x}\right]$,

$$
\begin{aligned}
\vec{F} & =\int_{a_{x}}^{b_{x}} \vec{f}(x) d x \\
& \approx \frac{b_{x}-a_{x}}{2 k_{x}} \vec{c}^{\top} \sum_{j=1}^{k_{x}} \vec{f}_{j}
\end{aligned}
$$

for $k_{x}$ uniform subintervals of $\left[a_{x}, b_{x}\right]$, with $n$ quadrature points in each subinterval. The vector of quadrature weights $\vec{c} \in \Re^{n \times 1}$ is given by

$$
\vec{c}=\left[c_{1}, c_{2}, \cdots, c_{n}\right]^{\top}
$$

At the $j^{\text {th }}$ subinterval, we define $\vec{f}_{j} \in \Re^{n \times m}$ as

$$
\overrightarrow{f_{j}}=\left[\vec{f}\left(x_{1, j}\right), \vec{f}\left(x_{2, j}\right), \cdots, \vec{f}\left(x_{n, j}\right)\right]^{\top},
$$

where the nodes

$$
x_{i j}=a_{x}+\frac{b_{x}-a_{x}}{2 k_{x}}\left(x_{i}+2 j-1\right), i=1: n, j=1: k_{x}
$$

are mapped from tabulated Gauss nodes $x_{i}$ on the standard interval $[-1,1]$. For the full macroscopic model, we require double integration, so we employ the composite $n$-point quadrature rule iteratively on $\vec{f}$ as a function of scalars $x$ and $y$

$$
\begin{aligned}
\bar{F} & =\int_{a_{y}}^{b_{y}} \int_{a_{x}}^{b_{x}} \vec{f}(x, y) d x d y \\
& \approx\left(\frac{b_{x}-a_{x}}{2 k_{x}}\right)\left(\frac{b_{y}-a_{y}}{2 k_{y}}\right) \sum_{j_{y}=1}^{k_{y}} \sum_{i=1}^{n} c_{i} \vec{c}^{\top} \sum_{j_{x}=1}^{k_{x}} \vec{f}_{j_{x}, i, j_{y}}
\end{aligned}
$$

where $k_{y}$ is the number of subintervals in $\left[a_{y}, b_{y}\right]$ and $c_{i}$ is the $i^{\text {th }}$ component of $\vec{c}$. The function $\vec{f}_{j_{x}, i, j_{y}} \in \Re^{n \times m}$ is evaluated at the $j_{x}^{t h} x$-subinterval and the $j_{y}^{\text {th }} y$-subinterval

$$
\vec{f}_{j_{x}, i, j_{y}}=\left[f\left(x_{1, j_{x}}, y_{1, j_{y}}\right), f\left(x_{2, j_{x}}, y_{2, j_{y}}\right), \cdots, f\left(x_{n, j_{x}}, y_{n, j_{y}}\right)\right]^{\top} .
$$

The $y$-nodes are given by

$$
y_{i j}=a_{y}+\frac{b_{y}-a_{y}}{2 k_{y}}\left(x_{i}+2 j-1\right), i=1: n, j=1: k_{y} .
$$

In our implementation, $\vec{f}_{j_{x}, i, j_{y}}$ corresponds to the integrand of (80) where the local strain solution $\bar{\varepsilon}\left(\sigma+\sigma_{e}, T ; \delta\right)$ is discretized over an interval of $m$ time steps. In addition, we take the finite integration intervals to correspond with 


\begin{tabular}{|c|l|}
\hline Nodes & Weights \\
\hline \hline$\frac{ \pm \sqrt{15-2 \sqrt{30}}}{\sqrt{35}}$ & $\frac{18+\sqrt{30}}{36}$ \\
\hline$\frac{ \pm \sqrt{15+2 \sqrt{30}}}{\sqrt{35}}$ & $\frac{18-\sqrt{30}}{36}$ \\
\hline \hline
\end{tabular}

Table 2. Four-point Gaussian quadrature nodes and weights on [-1,1].

$[\mu-q z, \mu+q z]$, where $\mu$ is the arithmetic mean and $q z$ is a multiple of the standard deviation for each distribution. For $q \geq 2$, the interval encompasses at least $95.4 \%$ of the probability. In our calculations, we use a four-point quadrature rule having the standard nodes and weights listed in Table 2. Finally, we note that the quadrature solves $(81)$ at $[(n-1) k+1]^{2}$ nodes and that the overall efficiency of the model algorithm primarily depends on the rapid computation of $\bar{\varepsilon}$.

\subsection{Simulations}

In this section, we demonstrate and analyze the material behavior predicted by the model. Specifically, we illustrate superelasticity, the shape memory effect, and heat transfer phenomena pertinent to thin-film SMAs. Table 3 summarizes the default parameters used in this section's simulations where we assume a rectangular SMA geometry. Additionally, we assume a material density of $\rho=6450 \mathrm{~kg} / \mathrm{m}^{3}$, corresponding to published values of polycrystalline NiTi wires. Since we do not demonstrate temperature-resistivity hysteresis, we do not specify the temperature coefficients of resistivity $\lambda_{A}^{e}$ and $\lambda_{M}^{e}$.

The mean local energy parameters $\Delta u=73.8 \mathrm{MJ} / \mathrm{m}^{3}, \Delta \eta=0.218 \mathrm{MJ} / \mathrm{m}^{3} \mathrm{~K}^{-1}$ are estimated from (18) and (25) given the parameters in Table $3, \sigma_{A}(353 \mathrm{~K})=$ $212 \mathrm{MPa}$, and $T_{e q}=338 \mathrm{~K}$. In solving the macroscopic model, we recalculate the estimates of $\Delta u$ and $\Delta \eta$ in this manner for each distributed value of $\delta$.

\subsubsection{Superelasticity}

Figure 12 illustrates superelasticity predicted by the model. The initial state is taken to be austenite at ambient temperature and a load is prescribed at a rate of $1 \mathrm{MPa} / \mathrm{s}$. In contrast to the abrupt transitions illustrated in Figure 12 for the local material behavior, the macroscopic model predicts gradual and asymmetric transitions. In addition to a nonuniform lattice structure, the internal temperature evolution is a source of the gradual transitions. During the transformation from austenite to detwinned martensite, transformation enthalpy is released. Some of the heat is transferred to the environment via convection while the remainder produces an increase in the internal temperature as shown in Figure 12. Therefore, the temperature-dependent loading 


\begin{tabular}{|c|c|c|}
\hline Physical & Value & Units \\
\hline$E_{A}, E_{M}$ & $22.4,13.7$ & $\mathrm{GPa}$ \\
\hline$\lambda_{A}, \lambda_{M}$ & $11.0,6.6$ & $10^{-6} / \mathrm{K}$ \\
\hline$c_{A}, c_{M}$ & $3.744,2.903$ & $\mathrm{MJ} / \mathrm{m}^{3} \mathrm{~K}^{-1}$ \\
\hline$V$ & 6903 & $\mathrm{~nm}^{3}$ \\
\hline$\tau$ & 1.1 & $\mathrm{~ms}$ \\
\hline Heat Transfer & & \\
\hline$\rho_{A}^{e}, \rho_{M}^{e}$ & 100,80 & $\mu \Omega \mathrm{cm}$ \\
\hline$\epsilon$ & 0.10 & \\
\hline$h_{c}$ & 10 & $\mathrm{~W} / \mathrm{m}^{2} \mathrm{~K}^{-1}$ \\
\hline Hysteresis & & \\
\hline$\varepsilon_{T}$ & 0.0248 & \\
\hline $\bar{\delta}, z_{\delta}$ & 152,35 & $\mathrm{MPa}$ \\
\hline$z_{\sigma}$ & 25 & $\mathrm{MPa}$ \\
\hline Geometric & & \\
\hline$L$ & 1.5 & $\mathrm{~cm}$ \\
\hline$d$ & 100 & $\mu \mathrm{m}$ \\
\hline$w$ & 1.8 & $\mathrm{~mm}$ \\
\hline \hline
\end{tabular}

Table 3. Mean local parameter values used by default in simulations. Changes from the default in particular simulations are noted in the text.

transformation stress increases during a transformation. The reverse process occurs for the unloading stress.

Figure 13 illustrates the temperature-dependent stress-strain hysteresis predicted by the model. We start with temperatures at which austenite is stable. At cooler temperatures, self-accommodating martensite $\left(50 \% \mathrm{M}^{+}, 50 \%\right.$ $\left.M^{-}\right)$is the initial phase and the stress-induced transformation corresponds to martensite detwinning. Agreeing with experimental observations in $[42,50]$, the yield stress from austenite to martensite is shown to increase as tem-
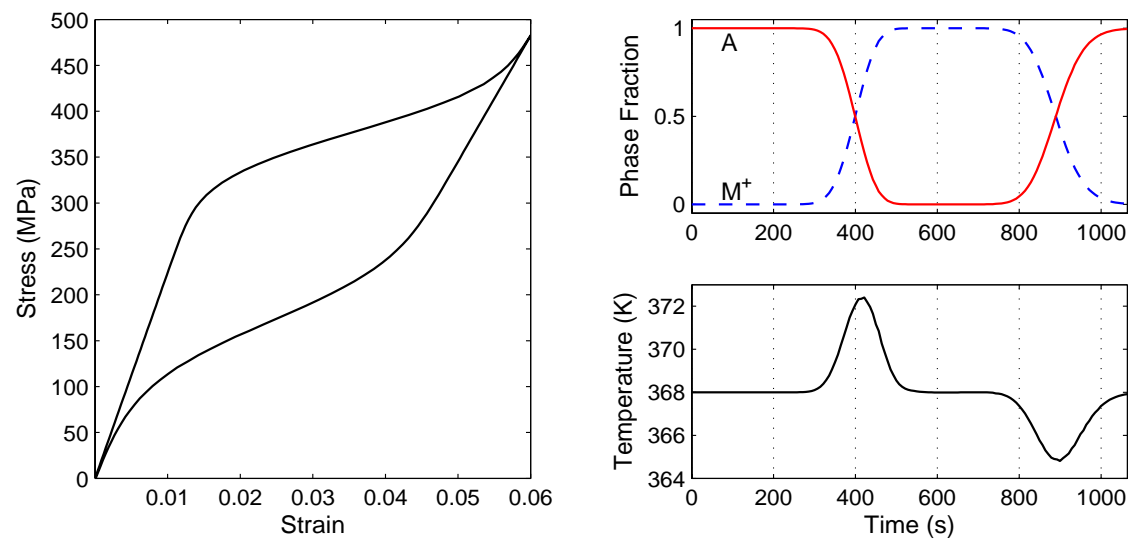

Fig. 12. Superelasticity at $T_{E}=358 \mathrm{~K}$. The release and absorption of transformation enthalpies yield internal temperature changes. 

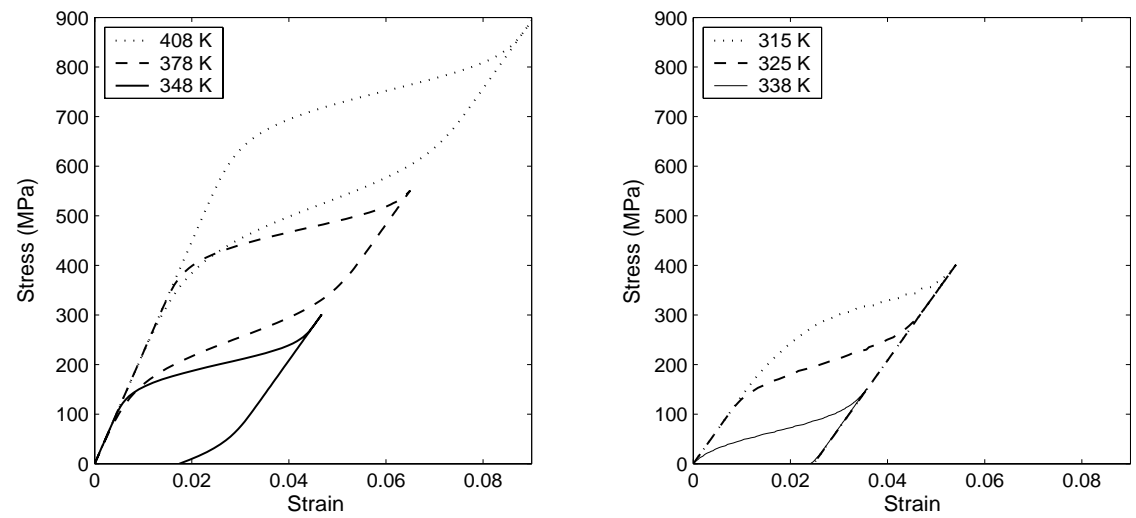

Fig. 13. The temperature-dependence of the hysteresis. For $T \geq 348 \mathrm{~K}$, austenite is the initial phase. Self-accommodating martensite is the initial phase for lower temperatures.

peratures increase, and the detwinning critical stress is shown to increase as temperatures decrease at lower temperatures. In Section 6.2, we compare the model to experimental superelasticity data.

\subsubsection{Shape Memory Effect}

Figure 14 illustrates the shape memory effect predicted by the model at a fixed positive load. The initial state is high-temperature austenite, and cooling and re-heating is achieved via convection by changing the external temperature at a rate of $0.1 \mathrm{~K} / \mathrm{s}$. As with superelasticity, the transformation enthalpies effect internal temperature changes, which in this case are small compared to the overall internal temperature. Additionally, while the local model describes steep transitions with distinct temperatures for the austenitemartensite transitions (see Figure 4), the macroscopic model predicts gradual
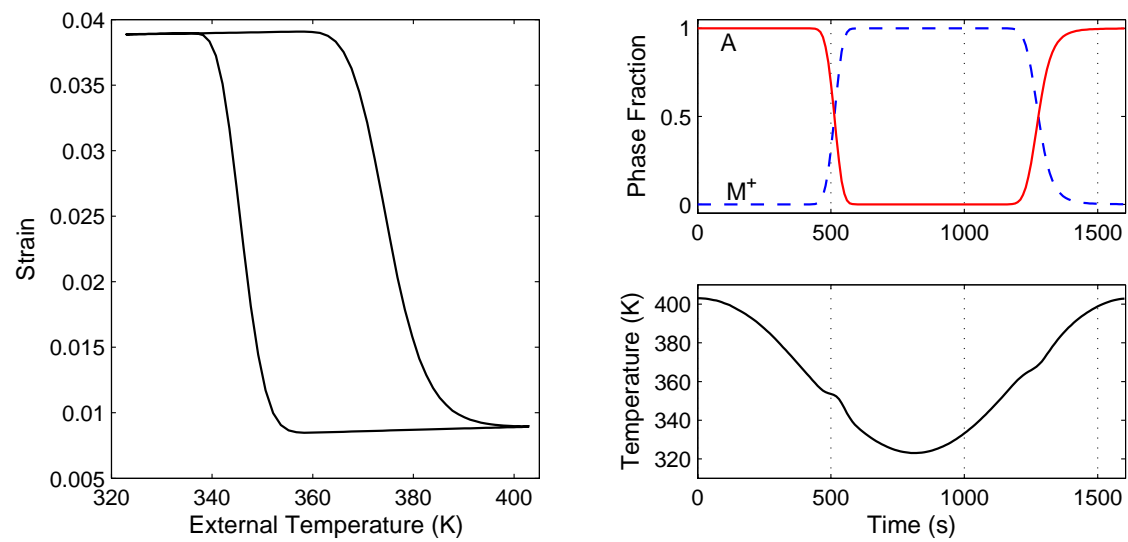

Fig. 14. Shape memory effect with a constant stress of $200 \mathrm{MPa}$. Cooling and heating is achieved by natural convection and the deviations of the temperature curve correspond with the release and absorption of transformation enthalpies. 
transitions where the beginning and end of the thermal transformations are not distinctly defined.

Figure 15 illustrates the stress-dependence of thermal hysteresis predicted by the model. The stress-dependent transformation temperatures are shown to decrease as stress decreases. Also, the transformation strain from austenite to detwinned martensite is shown to decrease rapidly at lower stresses. This experimentally verified behavior is attributed to the fact that low stresses are insufficient to induce a uniform transformation throughout the material (e.g., see $[31,51])$. The incorporation of effective stresses in (80) allows us to simulate this phenomenon and we note that other models are unable to account for this behavior $[15,31,88]$. In Section 6.3, we compare experimental SME data to model predictions.
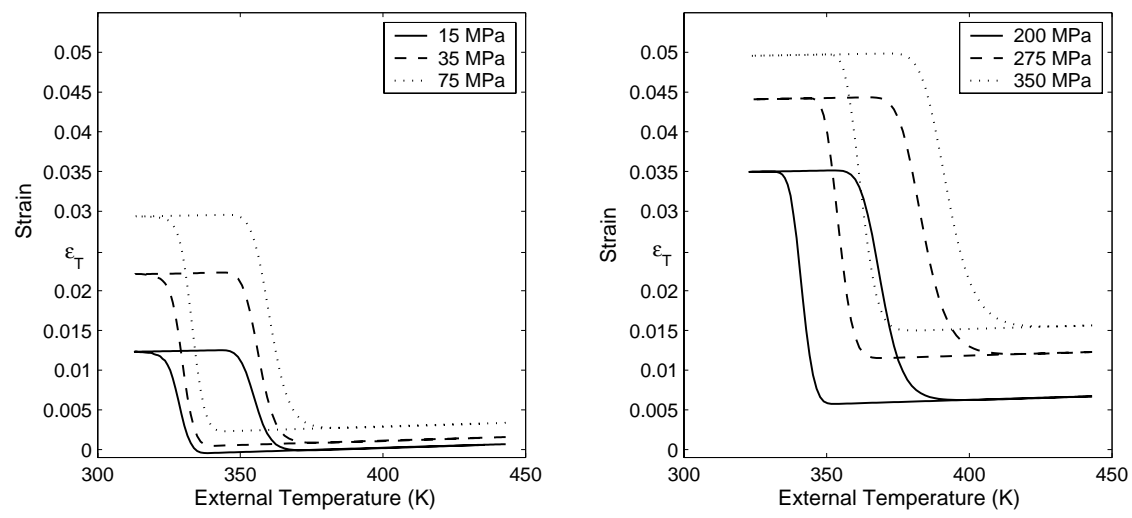

Fig. 15. The stress-dependence of thermal hysteresis. The initial phase is austenite at high temperatures and the low temperature phase is $M^{+}$.

\subsubsection{Heat Transfer Effects}

Here we investigate heat transfer aspects of the model with examples pertaining to thin-film SMA behavior. From (68), we see that the surface area to volume ratio of thin films is approximately $d^{-1}$. Accordingly, the effects of heat transfer will be prominent for thin films, which are 2-3 orders of magnitude thinner than typical bulk SMA wires and bars.

Figure 16 illustrates how thickness affects hysteresis in our model. Increased heat transfer due to increasing $\Omega$ allows latent heat to be quickly absorbed or released during transformations. Therefore, decreasing thicknesses yield less hysteresis, which allow for full actuation at smaller changes in stress and temperature.

In Figure 17, we demonstrate the actuation of a $10 \mu \mathrm{m}$ thin-film SMA via impulse Joule heating and forced convective cooling $\left(h_{c}=300 \mathrm{Wm}^{-2} / \mathrm{K}\right)$. The SMA is in a $303 \mathrm{~K}$ environment under a fixed stress of $100 \mathrm{MPa}$. Under these 

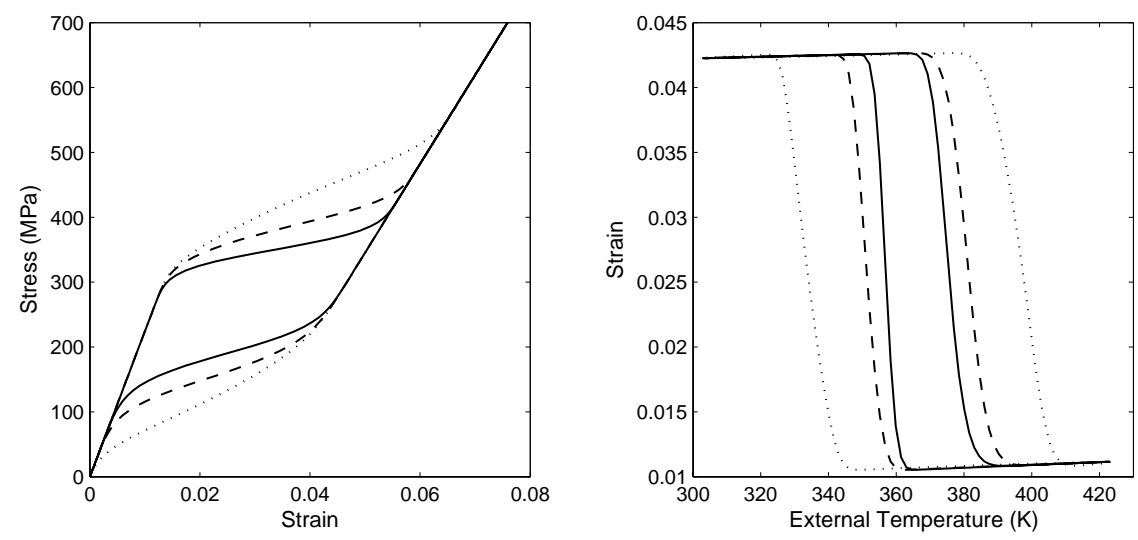

Fig. 16. Superelasticity $\left(T_{E}=358 \mathrm{~K}\right)$ and $\operatorname{SME}(250 \mathrm{MPa})$ for different film thicknesses. The solid, dashed, and dotted curves are for $d=10,100$, and $1000 \mu \mathrm{m}$, respectively. The standard deviations are taken to be $z_{\sigma}=20 \mathrm{MPa}$ and $z_{\delta}=20 \mathrm{MPa}$.

conditions, the SMA is deformed $3.2 \%$ as detwinned martensite. We simulated a low voltage of $2 \mathrm{~V}$ for a duration of $10 \mathrm{~ms}$, which increases the temperature by $50 \mathrm{~K}$ and induces a partial transformation to austenite with $1.2 \%$ recovered strain. A second and third impulse heating separated by $21 \mathrm{~ms}$ cooling times yields a full transformation to austenite and full deformation recovery. Finally, full reversion to martensite via forced convection is achieved in $50 \mathrm{~ms}$. The inner loop, which demonstrates that the model can maintain loop closure, corresponds to the first partial heating-cooling cycle.

From the temperature evolution in Figure 17, we conclude that cooling time in our model is the limiting factor for thin-film SMA actuator response. We also note the plateau in the temperature evolution. The delay in cooling corresponds with the phase transformation and is due to the exchange of transformation enthalpies. Similar analytical and experimental results are
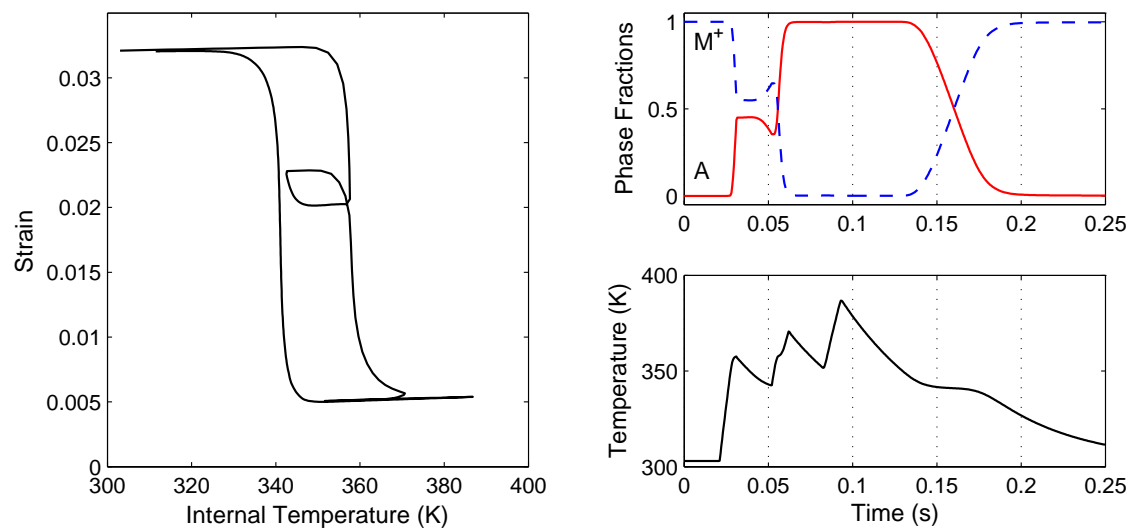

Fig. 17. Joule heating of a thin-film SMA and cooling via forced convection. The $10 \mu \mathrm{m}$ film is under a $100 \mathrm{MPa}$ stress and is initially detwinned martensite. The standard deviations are taken to be $z_{\sigma}=35 \mathrm{MPa}$ and $z_{\delta}=35 \mathrm{MPa}$. 
reported in $[69,87]$ and we note that $[73]$ concludes that the response of thinfilm SMA actuators is ultimately limited by the rate of phase transformations rather than the cooling time.

Finally, Figure 18 compares the temperature evolution for different values of $h_{c}$. In this case, $5 \mathrm{~V}$ is applied once for $3 \mathrm{~ms}$ to induce a full transformation. In each case, full recovery is achieved at approximately $320 \mathrm{~K}$, and the shortest total response time is $40 \mathrm{~ms}$.

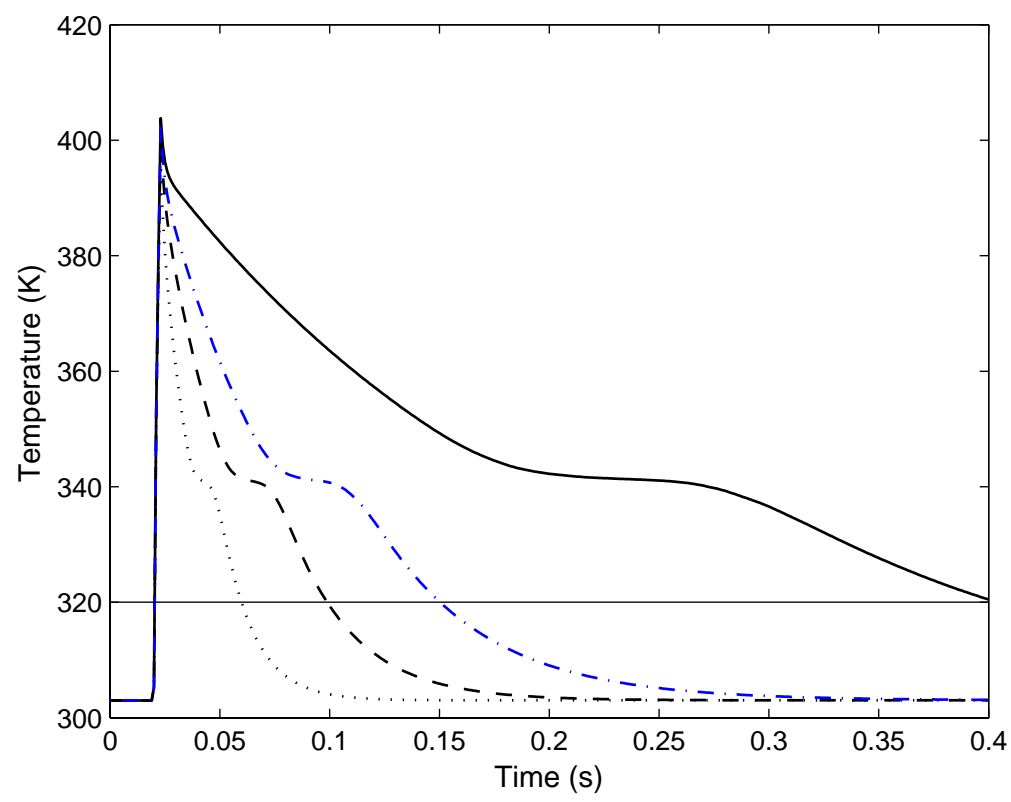

Fig. 18. The time response of a $10 \mu \mathrm{m}$ thin-film SMA. The solid, dash-dotted, dashed, and dotted curves respectively represent $h_{c}=100,300,500$, $1000 \mathrm{~W} / \mathrm{m}^{2} \mathrm{~K}^{-1}$. Complete reverse transformation occurs at $320 \mathrm{~K}$.

\section{Parameter Identification}

In this section, we discuss the identification of the model parameters in Table 1 via a combination of direct measurements and model calculations. The method of identification depends on the application under consideration as well as on the type of experimental data available. We describe three methods. The first pertains to stress-strain hysteresis, the second pertains to thermal hysteresis, and the third method pertains to direct measurements of material parameters without hysteresis data. Ultimately, a combination of these methods may be warranted for certain applications.

As illustrated in Figure 19, the macroscopic model (80) employs the local model as a kernel with certain parameters assumed to be manifestations of underlying distributions. Accordingly, we use known aspects of the local hysteresis model to obtain estimates of macroscopic material parameters from 

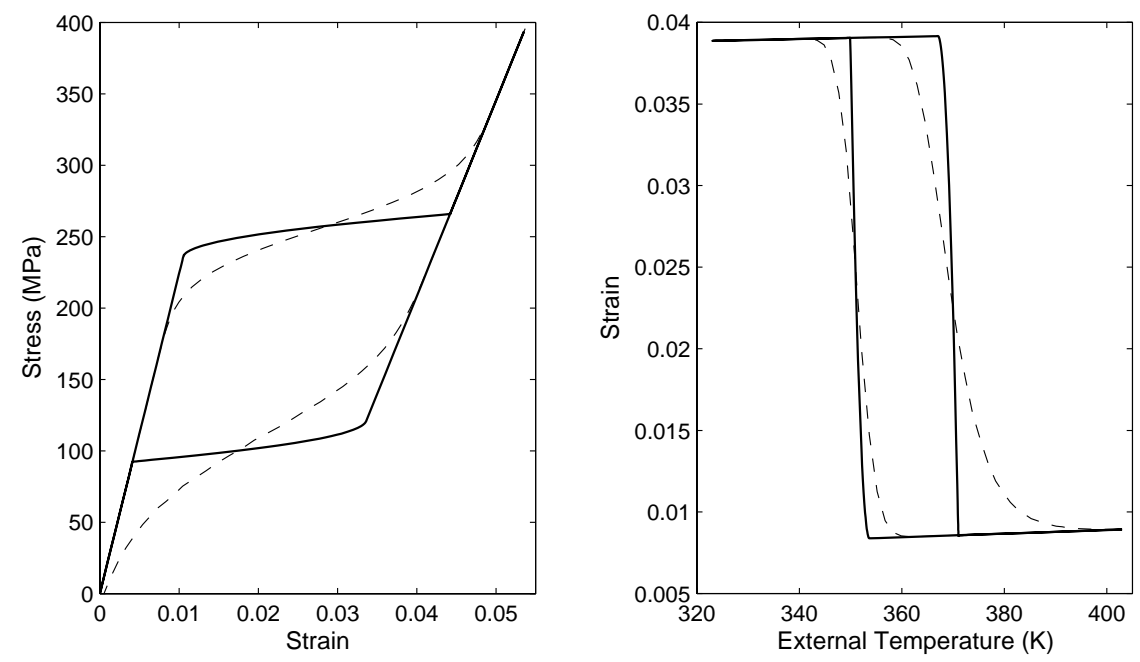

Fig. 19. The average local material response (solid) given by (59) compared with the statistically homogenized macroscopic response (dashed) given by (80).

measured hysteresis data. Estimates of the mean local parameters in Table 1 along with the distribution parameters, $z_{\delta}$ and $z_{\sigma}$ provide us initial parameter values for use in least squares fits to hysteresis data. We note that the activation volume $V$ and the relaxation time $\tau$ typically must be estimated from hysteresis data.

\subsection{Stress-strain Hysteresis}

One or more experimental stress-strain hysteresis curves are sufficient to estimate most of the material parameters pertaining to superelasticity. As illustrated in Figure 20, the elastic moduli can be estimated from linear portions of the loading or unloading curves. In particular, $E_{A}$ should be measured from linear portions of loading curves at sufficiently high temperatures. Similarly, $E_{M}$ can be measured from loading curves at relatively cool temperatures. Also at cool temperatures, $\varepsilon_{T}$ can be measured as the residual strain after full transformation detwinning [68]. Alternatively, both $E_{M}$ and $\varepsilon_{T}$ can be identified simultaneously by extrapolating a line from the superelastic unloading curve as depicted in Figure 20. Furthermore, $\bar{\delta}$ is estimated from the mean thickness of superelastic hysteresis.

With measurements of $E_{A}, E_{M}, \varepsilon_{T}$, and $\bar{\delta}$, the local relations (18), (25), (29), can be used to calculate the chemical free energy parameters $\Delta u, \Delta \eta$, and $\Delta c$ (recall $T_{R}=T_{e q}=\Delta u / \Delta \eta$ ). In this case, we also require the measurement of $\sigma_{A}$ or $\sigma_{M}$ at one or more temperatures as depicted in Figure 20. Therefore, if we make the first order approximation of $\Delta c=0$, only two stress-strain hysteresis curves at different temperatures are required to identify the local model fully for the prediction of superelasticity. Given the behavior illus- 


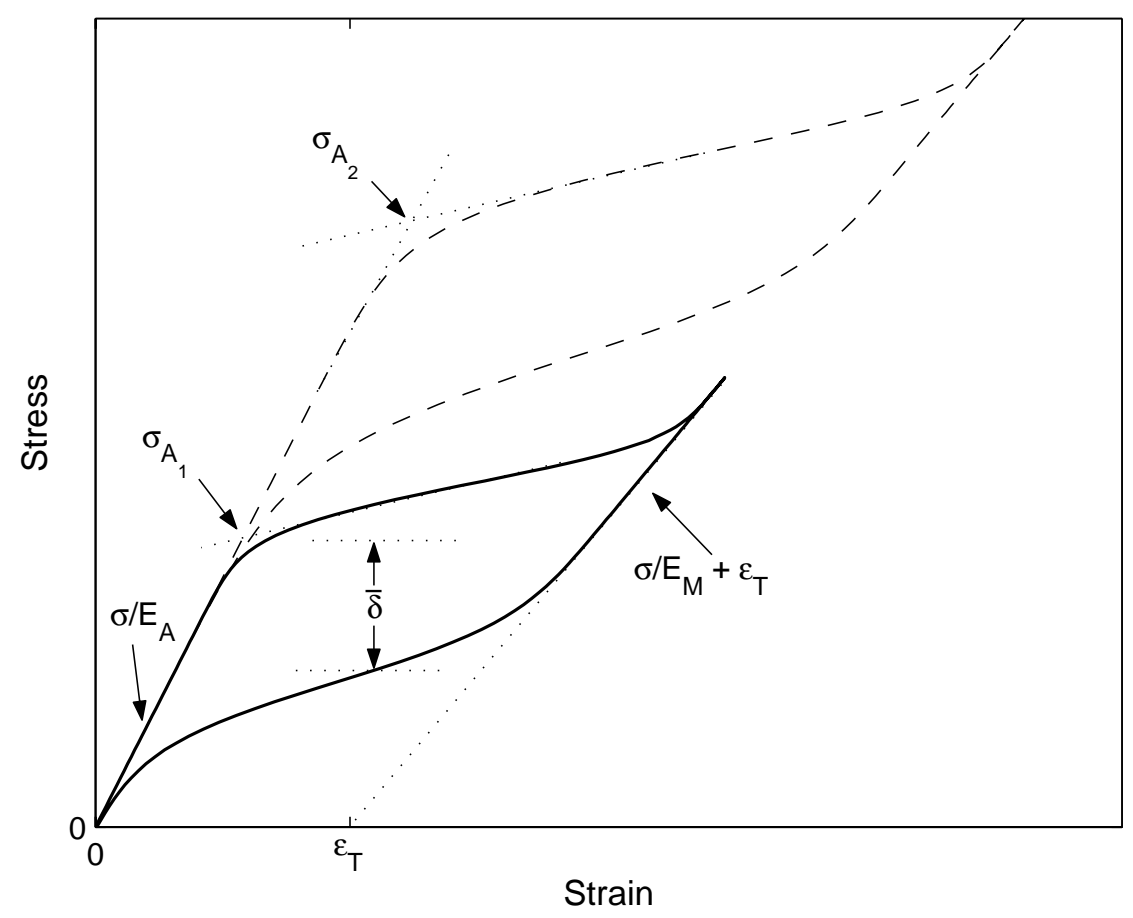

Fig. 20. Identification of material parameters using stress-strain hysteresis data. The dashed hysteresis curve corresponds to the higher ambient temperature $T_{2}>T_{1}$.

trated in Figure 19, we can estimate $z_{\delta}$ and $z_{\sigma}$ from the loading and unloading transformation regions.

We note that we have neglected to take into account heat transfer and rate phenomena described in Section 4.2 and we have implicitly assumed that the thermal activation effects described in Section 2.4 are insignificant. We take the identification methods we have described for near-isothermal, quasi-static cases as a first approximation for these cases. Finally, thermal expansion is normally negligible in superelasticity due to the small temperature changes. Nevertheless, the coefficients can be estimated using

$$
\lambda_{\alpha}=\frac{\varepsilon_{1}-\varepsilon_{2}}{T_{1}-T_{2}}
$$

where $\varepsilon_{i}$ are strains measured at the same stress level for temperatures $T_{i}$.

\subsection{Thermal Hysteresis}

Heat transfer effects are prominent in thermal hysteresis experiments; however, these effects can be minimized by slow heating rates, high rates of heat transfer, or direct measurements of the internal SMA temperature. One or more experimental temperature-strain hysteresis curves are sufficient to estimate most of the material parameters pertaining to the SME. The coefficients of thermal expansion can be estimated as the slopes of the extreme 
high-temperature (austenite) and low-temperature (martensite) portions of the hysteresis curve. As indicated in Figure 21, the elastic moduli can also be estimated from the extreme portions of the hysteresis curve. In particular, at a fixed stress $\sigma_{1}$

$$
E_{A}=\sigma_{1}\left[\varepsilon_{1}+\lambda_{A}\left(T_{1}-T_{0}\right)\right]^{-1},
$$

where $\varepsilon_{1}$ is the strain measured at temperature $T_{1}, T_{0}$ is the initial temperature of the cooling cycle, and $\lambda_{A}$ is the identified austenite thermal coefficient. In a similar manner, $E_{M}$ and $\varepsilon_{T}$ can be measured from the martensite portion of the hysteresis curve; however, as described in Section 8.2.2, this should be done at relatively high stresses so that the transformation in the material is complete. For the same temperature and two fixed stresses, we have

$$
E_{M}=\frac{\sigma_{2}-\sigma_{1}}{\varepsilon_{2}-\varepsilon_{1}}
$$

and

$$
\varepsilon_{T}=\frac{\sigma_{2} \varepsilon_{1}-\sigma_{1} \varepsilon_{2}}{\sigma_{2}-\sigma_{1}}
$$

Similar to the technique in [51], we can estimate the mean local transformation temperatures $T_{M}^{\sigma}$ and $T_{A}^{\sigma}$ as the average of the temperatures marking the beginning and end of transformations (see Figure 21). Finally, using the moduli, $\varepsilon_{T}$, and $T_{M}^{\sigma}$ and $T_{A}^{\sigma}$ at one or more stresses, we use (32) and (33) to determine $\Delta u, \Delta \eta, \Delta c$, and $\bar{\delta}$. We estimate $z_{\delta}$ and $z_{\sigma}$ from the gradual nature of the transformation temperature regions.

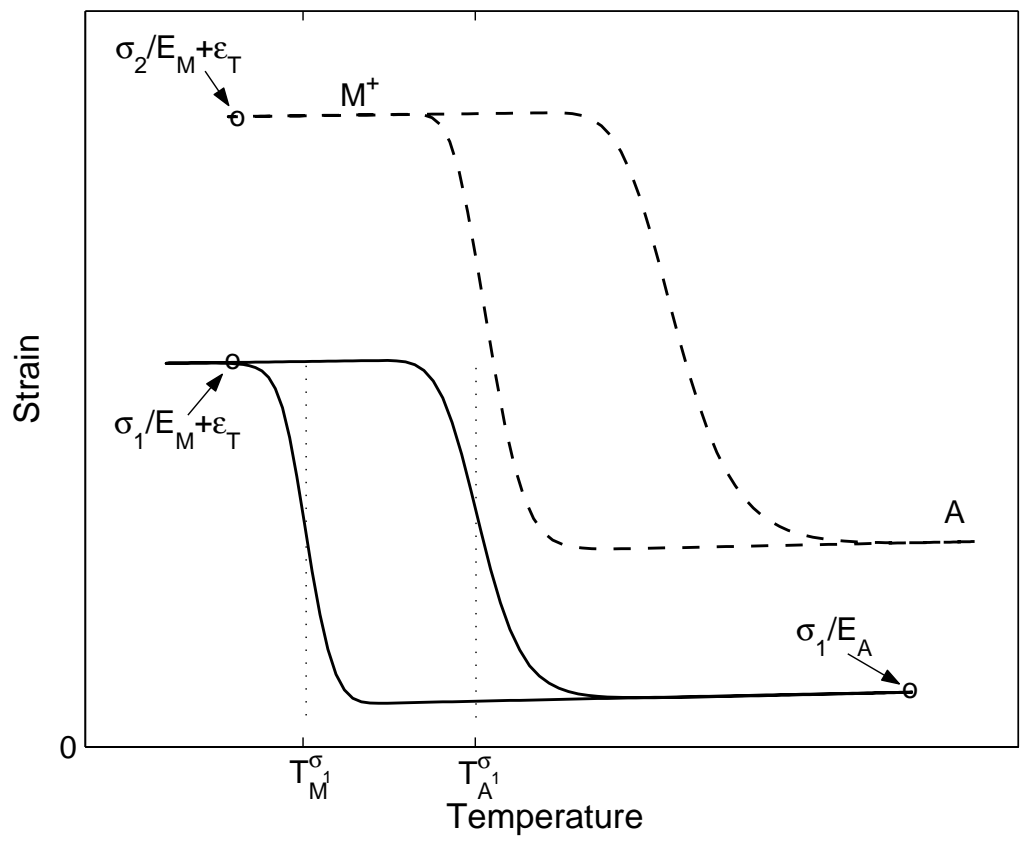

Fig. 21. Identification of material parameters using strain-temperature hysteresis data. The dashed hysteresis curve corresponds to the higher fixed stress $\sigma_{2}>\sigma_{1}$. 


\subsection{Non-hysteresis Measurements}

Other than direct hysteresis measurements, a number of material characterization methods have been developed for SMAs. We briefly summarize these methods. First, austenite and martensite elastic moduli are traditionally measured with non-cyclic, temperature-controlled tensile tests. However, when tensile tests are not feasible, as is typically the case for SMA thin films and microdevices, nanoindentation measurements are used [14,29,36,78,81].

In some cases, the chemical free energy parameters can be estimated directly. In particular, as described in [68], the difference in austenite and martensite reference entropies $\Delta \eta$ can be determined from differential scanning calorimetry (DSC) measurements, which are more commonly used to measure zero-stress transformation temperatures. One must take care to properly calibrate measurements to their chosen reference temperature $T_{R}$ in accordance with the model [100]. In addition to thermocouple techniques [27], DSC has also been used to measure phase-dependent specific heat capacities $[26,41,89,91,100,106]$. Furthermore, there are radiography techniques for measuring surface emissivity $\epsilon$; however, no results have been published for SMAs. We note that for aluminum film, $\epsilon=0.04$ and values for oxidized metals are closer to 0.5 [47]. Because of process impurities, oxidation of sputter-deposited thin-film SMA is often encountered during high-temperature annealing. In our treatment, we assume an emissivity of 0.1. Thermal expansion coefficients of SMAs have been measured using thermomechanical analyzers [107] (see Table 1 for published values). Finally, resistivities and their temperature coefficients are measured with four-point probes in a temperature-controlled chamber $[26,41,106]$. Next, we employ the parameter identification techniques described in this section to validate the model through comparison with experimental data.

\section{Experimental Validation}

In this section, we demonstrate the model's ability to simulate and predict measured material behavior. We provide three examples including anisothermal inner-loop superelasticity for a thin foil and superelasticity and SME for thin-film SMAs. In each case, we summarize the supplied data and material characterization results, identify parameters from the data, and compare the model predictions to hysteresis measurements.

\subsection{Inner Loops}

In this first example, we consider anisothermal stress-strain behavior of a NiTiFe foil manufactured by Furukawa Techno Material and tested in [77]. The foil was $500 \mu \mathrm{m}$ thick with $w=0.7 \mathrm{~cm}$ and $L=10 \mathrm{~cm}$ and had only

trace amounts of iron with a composition $50.5 \mathrm{at} . \% \mathrm{Ni}$ and $0.4 \mathrm{at} . \% \mathrm{Fe}$. The 
manufacturer-provided transformation temperatures were $A_{s}=234 \mathrm{~K}, A_{f}=$ $281 \mathrm{~K}, M_{s}=280 \mathrm{~K}, M_{f}=244 \mathrm{~K}$. The specimen was tested in an open air tensile machine $(293 \mathrm{~K}$ ambient) at a relatively fast strain rate of $0.01 / \mathrm{s}$ (equivalent to $12.67 \mathrm{MPa} / \mathrm{s}$ ). After 100 cycles of training, a multiple inner loop test was performed yielding the data shown in Figure 22.

The elastic moduli $E_{A}=37.2 \mathrm{GPa}$ and $E_{M}=25.5 \mathrm{GPa}$, and the transformation strain $\varepsilon_{T}=0.0253$ provided in [77] were measured from the bounding loop as discussed in Section 5.1. Additionally, we estimated $\bar{\delta}=70 \mathrm{MPa}$ and $\sigma_{A}(293 \mathrm{~K})=305 \mathrm{MPa}$ to calculate $T_{e q}=260 \mathrm{~K}$. No calorimetric analysis was available, so we estimated $c_{A}=c_{M}=2.9025 \mathrm{MJ} \mathrm{m}^{-3} \mathrm{~K}^{-1}$ from published data. Calorimetric results for a similar NiTi foil are reported in [91].

Using the bounding loop, we estimated the distribution parameters $z_{\delta}=$ $52 \mathrm{MPa}$ and $z_{\sigma}=55 \mathrm{MPa}$. For the activation energy volume, we took $V=$ $6903 \mathrm{~nm}^{3}\left(k_{B} / V=2 \mathrm{~J} \mathrm{~m}^{-3} \mathrm{~K}^{-1}\right)$ and chose a relaxation time $\tau=11.5 \mathrm{~ms}$. Finally, we used published NiTi values $\rho=6450 \mathrm{~kg} \mathrm{~m}^{-3}, \lambda_{A}=11.0 \times 10^{-6}$ $\mathrm{K}^{-1}$, and $\lambda_{M}=6.6 \times 10^{-6} \mathrm{~K}^{-1}$, and we estimated $h_{c}=10 \mathrm{~W} \mathrm{~m}^{-2} \mathrm{~K}^{-1}$ and $\epsilon=0.1$.

Using a load-controlled simulation, we compared the model with the identified parameters to the data in Figure 22. The hysteresis is highly sloped, most probably due to self-heating, corresponding to the fast strain rate. We note that while the model was fit to the outer bounding loop, it reasonably predicts the inner loop behavior.

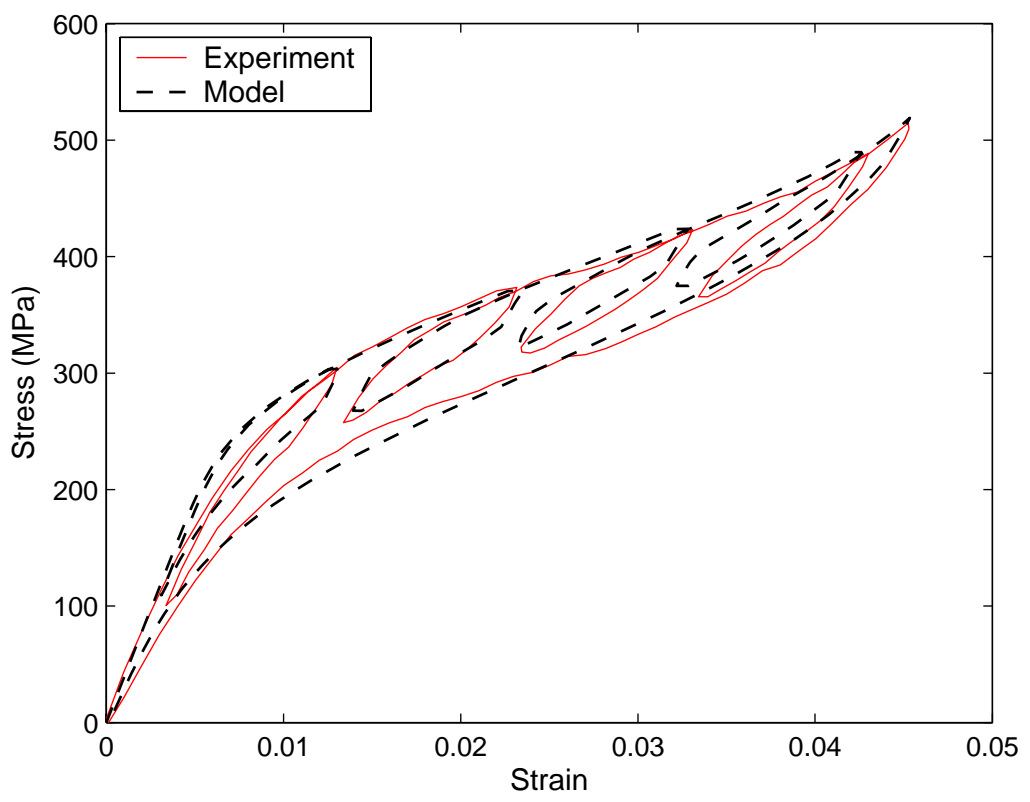

Fig. 22. The model compared to SMA stress-strain data. The inner loops are obtained successively off the loading path. Parameters were obtained through a fit to the bounding loop and the resulting model was used to predict the inner loops. 


\subsection{Superelasticity}

We consider here the near-isothermal stress-strain behavior for a thin-film SMA at different temperatures. A NiTi film of $8 \mu \mathrm{m}$ thickness was deposited onto a Si substrate using a dedicated DC magnetron sputtering system built at the UCLA Active Materials Lab [117]. After a 120 minute post-anneal at $773 \mathrm{~K}$, the start and finish transformation temperatures were measured with a DSC yielding $A_{s}=329 \mathrm{~K}, A_{f}=353 \mathrm{~K}, M_{s}=334 \mathrm{~K}, M_{f}=323 \mathrm{~K}$. Tensile specimens having the dimensions $w=0.1778 \mathrm{~cm}$ and $L=1.5 \mathrm{~cm}$ (gauge length) were cut from the film. Using an MTS microtensile apparatus, loadcontrolled $\left(0.94 \mathrm{MPa} \mathrm{s}^{-1}\right)$ tensile tests were performed in a thermal chamber. Two cyclic tests were performed yielding the data plotted in Figure 23.

The martensite modulus $E_{M}=13.7 \mathrm{GPa}$ and transformation strain $\varepsilon_{T}=$ 0.0352 were measured from an initial tensile test at $298 \mathrm{~K}$, and $E_{A}=22.4 \mathrm{GPa}$ and $\bar{\delta}=165 \mathrm{MPa}$ were estimated from a subsequent test at $353 \mathrm{~K}$. Furthermore, using $\sigma_{A}(353 \mathrm{~K})=220 \mathrm{MPa}$ and $-\sigma_{M}(298 \mathrm{~K})=105 \mathrm{MPa}$, we calculated $T_{e q}=313 \mathrm{~K}$ and specified $\Delta c=0.0636 \mathrm{MJ} \mathrm{m}^{-3} \mathrm{~K}^{-1}$. Further calorimetric analysis was not performed, so we estimated $c_{M}=2.9025 \mathrm{MJ} \mathrm{m}^{-3} \mathrm{~K}^{-1}$ from published data.

Using the superelastic data, we estimated the distribution parameters $z_{\delta}=$ $35 \mathrm{MPa}$ and $z_{\sigma}=10 \mathrm{MPa}$. For the activation energy volume, we took $V=$ $13807 \mathrm{~nm}^{3}\left(k_{B} / V=1 \mathrm{~J} \mathrm{~m}^{-3} \mathrm{~K}^{-1}\right)$ and chose a relaxation time $\tau=1.1 \mathrm{~ms}$. Finally, we used published NiTi values for $\rho=6450 \mathrm{~kg} \mathrm{~m}^{-3}$, we estimated $h_{c}=20 \mathrm{~W} \mathrm{~m}^{-2} \mathrm{~K}^{-1}$ corresponding with results in [69,87], $\epsilon=0.1$, and we neglected thermal expansion (near-isothermal conditions).

The model with the identified parameters is compared to the data in Figure 23. The SMA exhibits imperfect superelasticity at $353 \mathrm{~K}$ with a $0.18 \%$ resid-
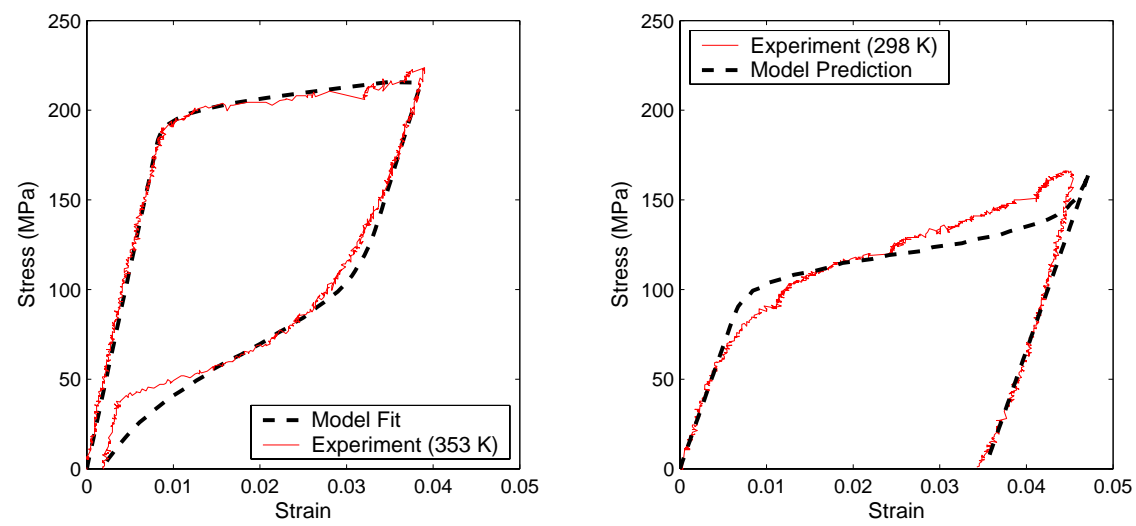

Fig. 23. The model compared to thin-film SMA stress-strain data. The superelastic curve at $353 \mathrm{~K}$ is used to fit model parameters and the behavior at $298 \mathrm{~K}$ is predicted by the model. 
ual strain. The model accurately predicts the asymmetric loading-unloading curves and the residual strain. At $298 \mathrm{~K}$, the observed transformation is from self-accommodating martensite to detwinned martensite. Using the fit to the superelastic data, we predicted the response at $298 \mathrm{~K}$. We note that both data correspond to partial cycles, as full transformations are not observed.

\subsection{Shape Memory Effect}

The final example we consider is the shape memory effect exhibited by a thinfilm SMA. Details of the fabrication and preparation of the test samples are provided in [51]. A near-equiatomic NiTi film of $9 \mu \mathrm{m}$ thickness was deposited onto a glass substrate using an RF magnetron sputtering system and was post annealed at $773 \mathrm{~K}$. Samples with dimensions of $w=1 \mathrm{~mm}$ and $L=5 \mathrm{~mm}$ were tested in a temperature-controlled microtensile tester. The SMA was loaded at high temperature (austenite) after which cooling and re-heating took place at $10 \mathrm{~K} / \mathrm{min}$ under a fixed load. A total of 18 cyclic tests were preformed at fixed stresses ranging from $20 \mathrm{MPa}$ to $600 \mathrm{MPa}$. Data from four of the tests were provided and are shown in Figure 24.

Since no calorimetric experiments were performed, we identified the model parameters directly from the hysteresis data. We used the 200, 360, and 520 $\mathrm{MPa}$ cases since it is likely the specimen did not fully transform at $40 \mathrm{MPa}$. We estimated the elastic moduli $E_{A}=41.331 \mathrm{GPa}$ and $E_{M}=20.313 \mathrm{GPa}$,
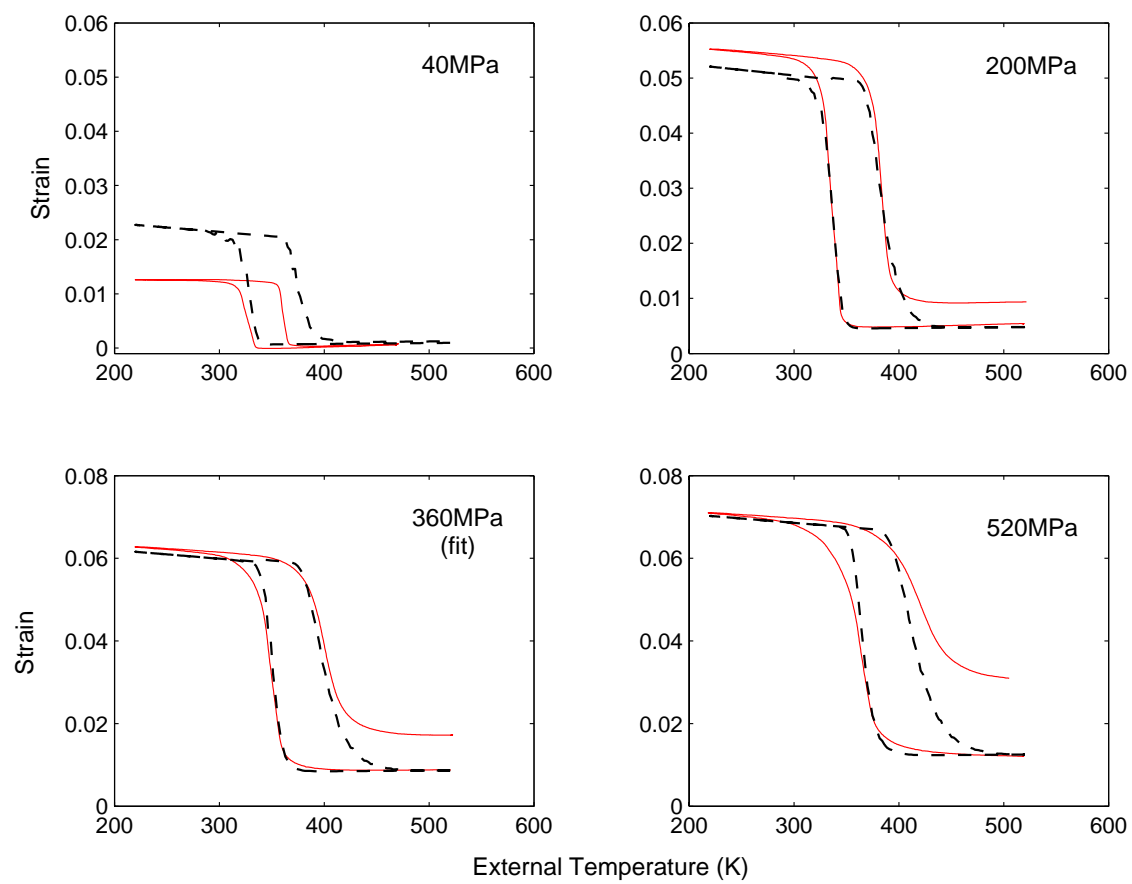

Fig. 24. The model (dashed) compared to thin-film SMA SME data (solid) at fixed stresses. The model is fit to the $360 \mathrm{MPa}$ data and predicts the other cases. 
and the transformation strain $\varepsilon_{T}=0.0388$ from linear fits. Furthermore, we measured $\lambda_{A}=1.7 \times 10^{-6} \mathrm{~K}^{-1}$ and $\lambda_{M}=-21 \times 10^{-6} \mathrm{~K}^{-1}$ from the 360 $\mathrm{MPa}$ data. Identifying $T_{M}^{\sigma}$ for each curve and $T_{A}^{\sigma}$ for the $360 \mathrm{MPa}$ case, we calculated $\bar{\delta}=530 \mathrm{MPa}, T_{e q}=344 \mathrm{~K}$ and $\Delta c=0.9966 \mathrm{MJ} \mathrm{m}^{-3} \mathrm{~K}^{-1}$.

We estimated the distribution parameters $z_{\delta}=40 \mathrm{MPa}$ and $z_{\sigma}=80 \mathrm{MPa}$. For the activation energy volume, we took $V=13807 \mathrm{~nm}^{3}\left(k_{B} / V=1 \mathrm{~J} \mathrm{~m}^{-3} \mathrm{~K}^{-1}\right)$ and chose a relaxation time $\tau=0.8 \mathrm{~ms}$. Finally, we used published NiTi values $\rho=6450 \mathrm{~kg} \mathrm{~m}^{-3}$ and $c_{M}=2.9025 \mathrm{MJ} \mathrm{m}^{-3} \mathrm{~K}^{-1}$ and we estimated $h_{c}=20 \mathrm{Wm}^{-2} \mathrm{~K}^{-1}$ and $\epsilon=0.1$.

The model is compared to the data in Figure 24. The SMA exhibits imperfect shape memory at higher loads due to accumulating plastic strains, which account for $32 \%$ of the transformation strain for the $520 \mathrm{MPa}$ test. While the model does predict a decrease in the effective transformation strain at lower stresses, it underestimates the strain at $200 \mathrm{MPa}$ and overestimates the strain at $40 \mathrm{MPa}$. Since we fit the model at $360 \mathrm{MPa}$ where the accumulated strain is 0.039 , it is likely our initial fit did not properly characterize the transformation strain of the material. Further thermal hysteresis tests should be considered where plastic deformation is not as significant.

\section{Concluding Remarks}

We have presented a model quantifying the macroscopic nonlinear behavior of shape memory alloys. In the first step of the development, we employed the framework of Müller-Achenbach-Seelecke to develop a local rate-dependent, thermomechanical model applicable to SMAs with uniform crystal lattices. Then we accommodated inhomogeneous and polycrystalline materials by averaging the variations of local material parameters and by considering nonuniform stress fields. The result is a rate-dependent, fully thermomechanical model that predicts relative elongation due to time-varying stress and temperature. We demonstrated that the model reasonably predicts the behavior of SMA films pertinent to actuator design such as superelasticity and the shape memory effect.

The model admits a low-order formulation making it viable for incorporation into engineering design applications and for implementation in model-based controllers. In particular, control analysis has been employed for analogous piezoceramic and magnetostrictive phase evolution models [80]. Moreover, the model provides a crucial step towards developing a unified methodology for modeling hysteresis in general ferroic materials [101].

The Boltzmann relations can yield a stiff ODE system in (81) and full coupling between stress and temperature effects may require additional distributions. 
Furthermore, statistical homogenization precludes the inverse problem of directly constructing new material compounds based on performance requirements.

There are opportunities for future research on the model. First, the development of control algorithms employing robust and multi-objective control frameworks should be considered. Second, following further validation, the model should be integrated into a finite element analysis infrastructure to support future design, performance evaluation, and qualification of thin-film SMA microdevices.

\section{Acknowledgments}

The authors would like to thank the following for providing the SMA hysteresis data used in this paper: Dr. Fuji Matsuzaki and Hisashi Naito at the Structural Dynamics Lab within Nagoya University in Nagoya, Japan; Dr. Greg P. Carman, Jason Woolman, and Dr. K.P. Mohachandra from the Active Materials Laboratory at UCLA in Los Angeles, CA; and Dr. Akira Ishida from the Materials Engineering Laboratory at the National Institute for Materials Science in Tsukuba, Japan. This research was supported in part by the National Science Foundation under the grant CMS-0099764 and by the Air Force Office of Scientific Research through the grant AFOSR-F49620-01-1-0107.

\section{References}

[1] R. Abeyratne and J.K. Knowles, "A continuum model of a thermoelastic solid capable of undergoing phase transitions," Journal of the Mechanics and Physics of Solids, 41(3), pp. 541-571, 1993.

[2] R. Abeyaratne, K. Bhattacharya and J.K. Knowles, "Strain-energy functions with multiple local minima: modeling phase transformations using finite thermoelasticity," Nonlinear Elasticity: Theory and Applications, pp. 433-490 (Ch. 12), Cambridge University Press, 2001.

[3] M. Achenbach, "A model for an alloy with shape memory," International Journal of Plasticity, Vol. 5, pp. 371-395, 1989.

[4] L.C. Andrews, Special Functions of Mathematics for Engineers, Second Ed., Ch. 3.2, McGraw-Hill, Inc., New York, USA, 1992.

[5] F. Auricchio and E. Sacco, "Thermo-mechanical modelling of a superelastic shape-memory wire under cyclic stretching-bending loadings," International Journal of Solids and Structures, 38(35), pp. 6123-6145, 2001.

[6] G.R. Barsch, "Landau theory of structural transformations in titanium-nickel and gold-cadmium alloys," Materials Science and Engineering: A, Vols. 273275, pp. 161-165, 1999. 
[7] G.R. Barsch, "Landau theory of the displacive phase transformations in goldcadmium and titanium-nickel alloys," Materials Science Forum, Vols. 327-328, pp. 367-376, 2000.

[8] P. Bĕlík and M. Luskin, "A computational model for the indentation and phase transformation of a martensitic thin film," Journal of the Mechanics and Physics of Solids, 50(9), pp. 1789-1815, 2002.

[9] Y. Bellouard, T. Lehnert, et. al., "Local annealing of complex mechanical devices: a new approach for developing monolithic micro-devices," Materials Science and Engineering: A, Vols. 273-275, pp. 795-798, 1999.

[10] Y. Bellouard, T. Lehnert, et. al., "Laser annealing of shape memory alloys: a versatile tool for developing smart micro-devices," Journal de Physique IV (Proceedings), 11(8), pp. 571-576, 2001.

[11] W.L. Benard, H. Kahn, et. al., "Thin-film shape-memory alloy actuated micropumps," Journal of Microelectromechanical Systems, 7(2), pp. 245-251, 1998.

[12] K. Bhattacharya and R.V. Kohn, "Symmetry, texture and the recoverable strain of shape-memory polycrystals," Acta Materialia, 44(2), pp. 529-542, 1996.

[13] K. Bhattacharyaa and R.D. James, "A theory of thin films of martensitic materials with applications to microactuators," Journal of the Mechanics and Physics of Solids, Vol. 47, pp. 531-576, 1999.

[14] A. Bhattacharyya, L. Sweeney and M.G. Faulkner, "Experimental characterization of free convection during thermal phase transformations in shape memory alloy wires," Smart Materials and Structures, 11(3), pp. 411$422,2002$.

[15] L.C. Brinson and M.S. Huang, "Simplifications and comparisons of shape memory alloy constitutive models," Journal of Intelligent Material Systems and Structures, Vol. 7, pp. 108-114, 1996.

[16] B. Bundara, M. Tokuda, et. al., "Superelastic tension and bending characteristics of shape memory alloys," Metals and Materials, 6(4), pp. 293$299,2000$.

[17] B. Bundara, "SMA: Present state and perspective for new applications," Materials Science Forum, Vols. 327-328, pp. 43-46, 2000.

[18] S. Büttgenbach, S. Bütefisch, et. al., "Shape memory microactuators," Microsystem Technologies, 7(4), pp. 165-70, 2001.

[19] T. Castán, E. Vives and A. Planes, "Elastic constants of BCC binary alloys near the $\mathrm{A}_{3} \mathrm{~B}$ composition and their relation to martensitic transitions," Journal of Physics: Condensed Matter, 2(7), pp. 1743-1752, 1990.

[20] C. Craciunescu and M. Wuttig, "Extraordinary damping of Ni-Ti double layer films," Thin Solid Films, 379(1-2), pp. 173-175, 2000. 
[21] R. DesRoches and M. Delemont, "Seismic retrofit of simply supported bridges using shape memory alloys," Engineering Structures, 24(3), pp. 325-332, 2002.

[22] T. Duerig, A. Pelton and D. Stöckel, "An overview of nitinol medical applications," Materials Science and Engineering: A, Vols. 273-275, pp. 149$160,1999$.

[23] F. Falk, "Landau theory of martensitic phase transitions," Journal de Physique Colloque, 43(C-4 ), pp. 3-15, 1982.

[24] F. Falk, "Pseudoelastic stress-strain curves of polycrystalline shape memory alloys calculated from single crystal data," International Journal of Engineering Science, 27(3), pp. 277-284, 1989.

[25] F. Falk, "Constitutive theories of shape memory alloys related to microstructure," Proceedings of the SPIE, Vol. 2442, pp. 2-10, 1995.

[26] M.G. Faulkner, J.J. Amalraj and A. Bhattacharyya, "Experimental determination of thermal and electrical properties of $\mathrm{Ni}-\mathrm{Ti}$ shape memory wires," Smart Materials and Structures, 9(5), pp. 632-639, 2000.

[27] J.E. Favelukis, A.S. Lavine and G.P. Carman, "An experimentally validated thermal model of thin-film NiTi," Smart structures and materials 1999: Smart Structures and Integrated Systems, Proceedings of the SPIE, Vol. 3668, Pt. 1-2, pp. 617-629, 1999.

[28] Y. Fu and H. Du, "Effects of film composition and annealing on residual stress evolution for shape memory TiNi film," Materials Science and Engineering A, Vol. 342, pp. 236-244, 2003.

[29] Y. Fu, W. Huang, et. al. "Characterization of TiNi shape-memory alloy thin films for MEMS applications," Surface and Coatings Technology, 145(1-3), pp. 107-112, 2001.

[30] H. Fujita and H. Toshiyoshi, "Micro actuators and their applications," 29(9), pp. 637-640, 1998.

[31] B. Gabry, C. Lexcellent, et. al., "Thermodynamic modeling of the recovery strains of sputter-deposited shape memory alloys $\mathrm{Ti}-\mathrm{Ni}$ and $\mathrm{Ti}-\mathrm{Ni}-\mathrm{Cu}$ thin films," Thin Solid Films, 372(1-2), pp. 118-133, 2000.

[32] V. Galhotra, V. Gupta, et. al., "Shape memory alloy based micro actuators," ACTUATOR 2000: 7th International Conference on New Actuators, pp. 338$341,2000$.

[33] W.S. Galinaitis, D.S. Joseph and R.C. Rogers, "Parameter identification for Preisach models of hysteresis," Proceedings of DETC'01: the ASME Design Engineering Technical Conference and Computers and Information in Engineering Conference, Vol. 6, Pt. B, pp. 1409-1417, 2001.

[34] K. Gall and H. Sehitoglu, "The role of texture in tension-compression asymmetry in polycrystalline NiTi," International Journal of Plasticity, 15(1), pp. 69-92, 1999. 
[35] K. Gall, H. Sehitoglu and Y. Chumlakov, "NiTi experiments versus modeling: Where do we stand?" Smart Structures and Materials 2000: Active Materials: Behavior and Mechanics, Proceedings of the SPIE, Vol. 3992, pp. 536-547, 2000.

[36] J.I. Gersten and F.W. Smith, The Physics and Chemistry of Materials, Ch. 4.6, John Wiley \& Sons, New York, USA, 2001.

[37] J. Gill, D. Chang, et. al., "Manufacturing issues of thin film NiTi microwrapper," Sensors and Actuators A, Vol. 93, pp. 148-156, 2001.

[38] J. Gill, K. Ho and G.P. Carman, "Three-dimensional thin-film shape memory alloy microactuator with two-way effect," Journal of Microelectromechanical Systems, 11(1), pp. 68-77, 2002.

[39] D.S. Grummon, S. Nam and L. Chang, "Effect of superelastically deforming NiTi surface microalloys on fatigue crack nucleation in copper," Shape Memory Materials and Phenomena - Fundamental Aspects and Applications Symposium, pp. 259-264, 1992.

[40] H.P. Ho, C.Y. Chung, et. al., "Novel far infrared imaging sensor based on the use of titanium-nickel shape memory alloys," Smart Structures, Devices, and Systems, Proceedings of the SPIE, Vol. 4935, pp. 69-73, 2002.

[41] L. Hou, "Processing, microstructure and thermomechanical behavior of superelastic nickel-titanium thin films sputter-deposited at elevated temperatures," Ph.D. Thesis, Dept. of Materials Science and Mechanics, Michigan State University, 1998.

[42] L. Hou and D.S. Grummon, "Transformational superelasticity in sputtered titanium-nickel thin films," Scripta Metallurgica, Vol. 33(6), pp. 989-995, 1995.

[43] L. Hou, T.J. Pence and D.S. Grummon, "Structure and thermal stability in titanium-nickel thin films sputtered at elevated-temperature on inorganic and polymeric substrates," Materials Research Society Symposium Proceedings, Vol. 360, pp. 369-374, 1995.

[44] W.M. Huang, J.P. Tan, et. al., "Design, testing, and simulation of NiTi shapememory-alloy thin-film-based microgrippers," Journal of Microlithography, Microfabrication, and Microsystems, 2(3), pp. 185-190, 2003.

[45] D. Hughes and J.T. Wen, "Preisach modeling of piezoceramic and shape memory alloy hysteresis," Smart Materials and Structures, 6(3), pp. 287-300, 1997.

[46] Y. Huo, "A mathematical model for the hysteresis in shape memory alloys," Continuum Mechanics and Thermodynamics, 1(4), pp. 283-303, 1989.

[47] F.P. Incropera and D.P. DeWitt, Fundamentals of Heat and Mass Transfer, Fifth Ed., John Wiley and Sons, Inc., New York, USA, 2002.

[48] A. Ishida and V. Martynov, "Sputter-deposited shape-memory alloy thin films: properties and applications," MRS-Bulletin, 27(2), pp. 111-114, 2002. 
[49] A. Ishida, M. Sato, et. al., "Bimorph-type microactuator using TiNi shapememory film," Materials Science Forum, Vols. 394-395, pp. 487-490, 2002.

[50] A. Ishida, M. Sato, et. al., "Stress-strain curves of sputter-deposited Ti-Ni thin films," Philisophical Magazine A, 80(4), pp. 967-980, 2000.

[51] A. Ishida, A. Takei, et. al., "Shape memory behavior of Ti-Ni thin films annealed at various temperatures," Materials Research Society Symposium Proceedings, Vol. 360, pp. 381-385, 1995.

[52] A.P. Jardine, J. Bartley-Cho and J. Flanagan, "Improved design and performance of the SMA torque tube for the DARPA Smart Wing Program." Smart structures and materials 1999. Industrial and commercial applications of smart structures technologies., Vol. 3674, pp. 260-269, 1999.

[53] Y.M. Jin and G.J. Weng, "Micromechanics study of thermomechanical characteristics of polycrystal shape-memory alloy films," Thin Solid Films, 376(1-2), pp. 198-207, 2000.

[54] A.D. Johnson, V. Martynov and V. Gupta, "Applications of shape memory alloys: advantages, disadvantages, and limitations," Micromachining and Microfabrication Process Technology VII, Proceedings of the SPIE, Vol. 4557, pp. 341-351, 2001.

[55] S. Kakac and Y. Yener, Convective Heat Transfer, Second Ed., CRC Press, Boca Raton, USA, 1995.

[56] T. Kim, Q. Su and M. Wuttig, "Thermo-mechanical $\mathrm{Ni}_{50} \mathrm{Ti}_{50} / \mathrm{Si}$ composite thin film switch," Materials Research Society Symposium Proceedings, Vol. 360, pp. 375-380, 1995.

[57] M. Kohl, D. Dittmann, et. al., "Shape memory microvalves based on thin films or rolled sheets," Materials Science and Engineering A, Vols. 273-275, pp. 784-788, 1999.

[58] M. Kohl, D. Dittmann, et. al., "Thin film shape memory microvalves with adjustable operation temperature," Sensors and Actuators A: Physical, 83(13), pp. 214-219, 2000.

[59] M. Kohl, B. Krevet and E. Just, "SMA microgripper system," Sensors and Actuators A: Physical, Vols. 97-98, pp. 646-652, 2002.

[60] M. Kohl and K.D. Skrobanek, "Linear microactuators based on the shape memory effect," Sensors and Actuators A: Physical, 70(1-2), pp. 104-111, 1998.

[61] A.R. Krommer and C.W. Ueberhuber, Computational Integration, Ch. 5, SIAM, Philadelphia, USA, 1998.

[62] E. Kröner, "Statistical Modeling," in Modelling Small Deformations of Polycrystals, edited by J. Gittus and J. Zarka, pp. 229-291, 1986.

[63] P. Krulevitch, A.P. Lee, et. al., "Thin film shape memory alloy microactuators," Journal of Microelectromechanical Systems, 5(4), pp. 270$282,1996$. 
[64] A. Ktena, D.I. Fotiadis, P.D. Spanos, et. al., "A Preisach model identification procedure and simulation of hysteresis in ferromagnets and shape-memory alloys," Physica B: Condensed Matter, 306(1-4), pp. 84-90, 2001.

[65] K. Kuribayashi, "Micro SMA actuator and motion control," MHS2000. Proceedings of 2000 International Symposium on Micromechatronics and Human Science, pp. 35-42, 2000.

[66] K. Kuribayashi and T. Fujii, "A new micro SMA thin film actuator prestrained by polyimide," MHA'98. Proceedings of the 1998 International Symposium on Micromechatronics and Human Science, pp. 165-170, 1998.

[67] D.C. Lagoudas and A. Bhattacharyya, "On the correspondence between micromechanical models for isothermal pseudoelastic response of shape memory alloys and the Preisach model for hysteresis," Mathematics 85 Mechanics of Solids, 2(4), pp. 405-440, 1997.

[68] D.C. Lagoudas and Z. Bo, "Thermomechanical modeling of polycrystalline SMAs under cyclic loading, Part II: material characterization and experimental results for a stable transformation cycle," International Journal of Engineering Science, 37(9), pp. 1141-1173, 1999.

[69] A.S. Lavine and G.P. Carman, "Thermomechanical Modeling of Thin Film Shape Memory Alloy Devices," Thermal Science and Engineering, 8(4), pp. 13$25,2000$.

[70] A.P. Lee, D.R. Ciarlo, et. al., "A practical microgripper by fine alignment, eutectic bonding and SMA actuation," Sensors and Actuators A: Physical, 54(1-3), pp. 755-759, 1996.

[71] C. Lexcellent, S. Moyne, et. al., "Deformation behaviour associated with the stress-induced martensitic transformation in $\mathrm{Ti}-\mathrm{Ni}$ thin films and their thermodynamical modelling," Thin Solid Films, 324(1-2), pp. 184-189, 1998.

[72] E. Limpert, W.A. Stahel and M. Abbt, "Log-normal Distributions across the Sciences: Keys and Clues," Bioscience, 51(5), pp. 341-352, (2001).

[73] C.C. Ma, R. Wang, et. al., "Frequency response of TiNi shape memory alloy thin film micro-actuators," Proceedings of IEEE, the Thirteenth Annual International Conference on Micro Electro Mechanical Systems, pp. 370-374, 2000 .

[74] E. Makino, T. Mitsuya and T. Shibata, "Dynamic actuation properties of TiNi shape memory diaphragm," Sensors and Actuators A: Physical, 79(2), pp. 128-135, 2000.

[75] V. Martynov, "TiNi thin films for microactuators and microdevices: Sputter deposition and processing techniques," Materials Science Forum, Vols. 426432, pp. 3475-3480, 2003.

[76] J.E. Massad and R.C. Smith, "A Domain Wall Model for Ferroelastic Materials," Journal of Intelligent Material Systems and Structures, 7(7), pp. 455-471, 2003. 
[77] Y. Matsuzaki, K. Funami and H. Naito, "Inner loops of pseudoelastic hysteresis of shape memory alloys: Preisach approach," Smart Structures and Materials 2002: Active Materials: Behavior and Mechanics, Proceedings of the SPIE, Vol. 4699, pp. 355-364, 2002.

[78] S. Miyazaki and A. Ishida, "Martensitic transformation and shape memory behavior in sputter-deposited TiNi-based thin films," Materials Science and Engineering A, Vols. 273-275, pp. 106-133, 1999.

[79] K. Mori, H. Li, et. al., "Patterned shape memory alloy films," Materials Transactions, 43(5), pp. 951-955, 2002.

[80] J.M. Nealis and R.C. Smith, "Control techniques for high performance nonlinear smart systems," Smart Structures and Materials 2003, Proceedings of the SPIE, Vol. 5049, pp. 221-232, 2003.

[81] W. Ni and D.S. Grummon, "Nanoindentation and wear behavior of nickeltitanium alloys and thin films," Materials Research Society Symposium Proceedings, Vol. 697, pp.53-58, 2002.

[82] K. Otsuka and C.M. Wayman, Shape Memory Materials, Cambridge University Press, Cambridge, United Kingdom, 1998.

[83] N. Papenfuß and S. Seelecke, "Simulation and control of SMA actuators," Smart structures and materials 1999: Mathematics and control in smart structures, Proceedings of the SPIE, Vol. 3667, pp. 586-595, 1999.

[84] E. Patoor and M. Berveiller, "Micromechanical modeling of the thermomechanical behavior of shape memory alloys," Mechanics of Solids with Phase Changes, pp. 121-188, 1997.

[85] J. Peirs, D. Reynaerts and H. Van-Brussel, "Scale effects and thermal considerations for micro-actuators," Proceedings, 1998 IEEE International Conference on Robotics and Automation, Vol. 2, pp. 1516-1521, 1998.

[86] R. Philips, Crystals, Defects and Microstructures: Modeling Across Scales, Ch. 7.4.2, Cambridge University Press, Cambridge, UK, 2001.

[87] P.L. Potapov and E.P. Da Silva, "Time response of shape memory alloy actuators," Journal of Intelligent Material Systems and Structures, 11(2), pp. 125-134, 2000.

[88] H. Prahlad and I. Chopra, "Comparative evaluation of shape memory alloy constitutive models with experimental data," Journal of Intelligent Material Systems and Structures, 12(6), pp. 383-394, 2001.

[89] H. Prahlad and I. Chopra, "Development of a strain-rate dependent model for uniaxial loading of SMA wires," Journal of Intelligent Material Systems and Structures, 14(7), pp. 429-442, 2003.

[90] I. Roch, Ph. Bidaud, "Fabrication and characterization of an SU-8 gripper actuated by a shape memory alloy thin film," Journal of Micromechanics and Microengineering, 13(2), pp. 330-336, 2003. 
[91] M. Rohde and A. Schüssler, "On the response-time behaviour of laser micromachined NiTi shape memory actuators," Sensors and Actuators A: Physical, 61(1-3), pp. 463-468, 1997.

[92] S. Saadat, M. Noori, et. al., "Using NiTi SMA tendons for vibration control of coastal structures," Smart Materials and Structures, 10(4), pp. 695-704, 2001.

[93] B. Schroeder, C. Boller, et. al., "Comparative assessment of models for describing the constitutive behaviour of shape memory alloys," Smart materials and structures: proceedings of the 4th European Conference on Smart Structures and Materials in conjunction with the 2nd International Conference on Micromechanics, Intelligent Materials, and Robotics, pp. 305$312,1998$.

[94] S. Seelecke and I. Müller, "Shape memory alloy actuators in smart structures - modeling and simulation," ASME Applied Mechanics Reviews, to appear, 2003.

[95] J.A. Shaw and S. Kyriakides, "Thermodynamic aspects of NiTi," Journal of the Mechanics and Physics of Solids, 43(8), pp. 1243-1281, 1995.

[96] J.A. Shaw, "A thermomechanical model for a 1-D shape memory alloy wire with propagating instabilities," International Journal of Solids and Structures, 39(5), pp. 1275-1305, 2002.

[97] D.D. Shin, G.P. Carman, "Operating frequency of thin film NiTi in fluid media," Proceedings of 2001 ASME IMECE, MEMS Symposium, pp. 221-227, 2001.

[98] D.D. Shin, K.P. Mohanchandra, et. al., "Miniature actuator using thin-film shape-memory alloy," Smart Structures and Materials 2003: Industrial and Commercial Applications of Smart Structures Technologies, Proceedings of the SPIE, Vol. 5054, pp. 118-124, 2003.

[99] Y. Shu, "Shape-memory micropumps," Materials Transactions, 43(5), pp. 1037-1044, 2002.

[100] J.F. Smith, Q. Jiang, et. al., " $\mathrm{C}_{p}$ and fractal phase transformation in the shape memory alloy $\mathrm{Ni}-52 \mathrm{Ti}$," Materials Science and Engineering A, 149(1), pp. 111-120, 1991.

[101] R.C. Smith, S. Seelecke, et. al., "A unified model for hysteresis in ferroic materials," Smart Structures and Materials 2003: Modeling, Signal Processing, and Control, Proceedings of the SPIE, Vol. 5049, pp. 88-99, 2003.

[102] T. Sterzl, B. Winzek, et. al., "Bistable shape memory thin film actuators," Smart Structures and Materials 2003: Active Materials: Behavior and Mechanics, Proceedings of the SPIE, Vol. 5053, pp. 101-109, 2003.

[103] V. Stoilov and A. Bhattacharyya, "A theoretical framework of one-dimensional sharp phase fronts in shape memory alloys," Acta Materialia, 50(20), pp. 4939$4952,2002$. 
[104] M. Tabib-Azar, B. Sutapun and M. Huff, "Applications of TiNi thin film shape memory alloys in micro-opto-electro-mechanical systems," Sensors and Actuators A: Physical, 77(1), pp. 34-38, 1999.

[105] H. Tamai and Y. Kitagawa, "Pseudoelastic behavior of shape memory alloy wire and its application to seismic resistance member for building," Computational Materials Science, 25(1-2), pp. 218-227, 2002.

[106] J. Uchil, K.P. Mohanchandra, et. al., "Study of critical dependence of stable phases in Nitinol on heat treatment using electrical resistivity probe," Materials Science and Engineering A, Vol. 251, pp. 58-63, 1998.

[107] J. Uchil, K.P. Mohanchandra, et. al., "Thermal expansion in various phases of Nitinol using TMA," Physica B, Vol. 270, pp.289-297, 1999.

[108] A. Visitin, Differential Models of Hysteresis, Springer-Verlag, New York, 1994.

[109] R.X. Wang, Y. Zohar and M. Wong, "The effects of process-induced stress on the microstructures and the phase transformation characteristics of sputtered titanium-nickel thin-film shape-memory alloys," Journal of Micromechanics and Microengineering, 11(6), pp. 686-691, 2001.

[110] R.X. Wang, Y. Zohar and M. Wong, "Residual stress-loaded titanium-nickel shape-memory alloy thin-film micro-actuators," Journal of Micromechanics and Microengineering, 12(3), pp. 323-327, 2002.

[111] F. Warkusz, "The size effect and the temperature coefficient of total resistance in thin metal films," Journal of Physics D-Applied Physics, Vol. 11, 1978.

[112] G. Webb, A. Kurdila and D. Lagoudas, "Adaptive hysteresis model for model reference control with actuator hysteresis," Journal of Guidance, Control, and Dynamics, 23(3), pp. 459-465, 2000.

[113] K. Wilde, P. Gardoni and Y. Fujino, "Base isolation system with shape memory alloy device for elevated highway bridges," Engineering Structures, 23(3), pp. 222-229, 2000.

[114] B. Winzek, T. Sterzl, et. al., "Smart motion control by phase-coupled shape memory composites," Proceedings of the 2002 IEEE/RSJ International Conference on Intelligent Robots and Systems, Vols. 1-3, pp. 2004-2009, 2002.

[115] B. Winzek, S. Schmitz, et. al., "SMA-thin film composites providing traveling waves," Smart structures and materials 2003: Active Materials: Behavior and Mechanics, Proceedings of the SPIE, Vol. 5053, pp. 110-118, 2003.

[116] D. Wood, J.S. Burdess and A.J. Harris, "Actuators and their mechanisms in microengineering," Engineering Science and Education Journal, 7(1), pp. 19-27, 1998.

[117] J.L. Woolman, K.P. Mohanchandra and G.P. Carman, "Composition and annealing effects on the mechanical properties of superelastic thin film nickel titanium," Smart Structures and Materials 2003, Proceedings of the SPIE, Vol. 5053, pp. 230-238, 2003. 
[118] M.R. Wuttig, "Thin film SMA/Si composite actuators ," Smart Structures and Materials 2000: Mathematics and Control in Smart Structures, Proceedings of the SPIE, Vol. 3984, pp. 450-455, 2000.

[119] D. Xu, L. Wang, et. al., "Characteristics and fabrication of NiTi/Si diaphragm micropump," Sensors and Actuators A: Physical, 93(1), pp. 87-92, 2001.

[120] Y.Y. Ye, C.T. Chan and K.M. Ho, "Structural and electronic properties of the martensitic alloys TiNi, TiPd, and TiPt," Physical Review B, 56(7), pp. 3678-3689, 1997.

[121] H. Zhang, Y. Bellouard, et. al., "A monolithic shape memory alloy microgripper for 3-D assembly of tissue engineering scaffolds," Microrobotics and Microassembly III, Proceedings of the SPIE, Vol. 4568, pp. 50-60, 2001. 